\title{
A neuroendocrine feedback loop in C. elegans males integrates food detection and biological sex to modulate chemoreceptor expression and behavioral flexibility
}

Leigh R. Wexler ${ }^{1}$, Renee M. Miller ${ }^{2}$, and Douglas S. Portman ${ }^{1,3,4,5 *}$

Departments of ${ }^{1}$ Biomedical Genetics, ${ }^{3}$ Neuroscience, and ${ }^{4}$ Biology

Department of ${ }^{2}$ Brain and Cognitive Sciences

${ }^{5}$ Ernest J. Del Monte Institute for Neuroscience

University of Rochester

Rochester, NY 14642

*Lead Contact and Corresponding Author: douglas.portman@rochester.edu

Keywords: $C$. elegans, feeding, chemosensation, behavioral choice, statedependence, starvation, sex differences, insulin signaling, TGF $\beta$ signaling 


\section{SUMMARY}

Dynamic integration of internal and external cues is essential for flexible, adaptive

3 animal behavior. In C. elegans, biological sex and feeding state regulate expression of the food-

4 associated chemoreceptor odr-10, contributing to plasticity in food detection and the decision

5 between feeding and exploration. In adult hermaphrodites, odr-10 expression is high; in well-

6 fed adult males, odr-10 expression is low, promoting exploratory mate-searching behavior.

7 Food-deprivation transiently activates male odr-10 expression, heightening food sensitivity and

8 reducing food-leaving. Here, we identify a neuroendocrine feedback loop that sex-specifically

9 regulates odr-10 in response to food deprivation. In well-fed males, insulin-like (IIS) and TGF $\beta$

10 signaling repress odr-10 expression. Upon food deprivation, odr-10 is directly activated by DAF-

11 16/FoxO, the canonical C. elegans IIS effector. The TGF $\beta$ ligand DAF-7 acts upstream of IIS, and,

12 likely because of its sexually dimorphic expression in the nervous system, links feeding to odr-

1310 only in males. Surprisingly, these responses to food deprivation are not triggered by internal

14 metabolic cues, but rather by the loss of sensory signals from food. In the presence of inedible

15 food, males become metabolically starved but express levels of odr-10 and daf-7 comparable to

16 those of well-fed males. Further, exposing food-deprived males to inedible food is sufficient to

17 restore low odr-10 expression. Food signals are detected by a small number of sensory neurons

18 whose activity non-autonomously regulates daf-7 expression, IIS, and odr-10. Thus, adult $C$.

19 elegans males employ a neuroendocrine feedback loop that integrates food detection and

20 genetic sex to dynamically modulate chemoreceptor expression and sensory behavior. 
Innate drives - like those to feed, reproduce, and avoid predators-provide a flexible

24 and modular framework for the control of animal behavior. Internal and external signals continuously interact with such drives, allowing behaviors to be activated or suppressed according to an animal's history, physiology, and environment. In humans, disruption of these

27 mechanisms is associated with a variety of neuropsychiatric conditions, including eating

28 disorders, depression, and obsessive-compulsive disorder. Though even the simplest nervous

29 systems employ flexible, innate drives, much remains unknown about how they are regulated by internal and external conditions, how these conditions are represented internally, and how drive states influence behavioral choice.

33 modulation of sensory perception. Feeding state is a primary source of such regulation: for example, hunger and satiety modulate chemosensory function in Drosophila [1-4], C. elegans [5-12], and mice $[13,14]$, as well as mechanosensation in the leech [15]. In this way, hunger focuses an animal's attention on food-related appetitive stimuli and attenuates the perception

37 of cues that might otherwise inhibit feeding $[16,17]$. In most of these cases, feeding state information is thought to originate from internally derived metabolic cues, with the role of acute sensory input in influencing internal state being less well appreciated.

41 influence sensory function [18]. In C. elegans, for example, biological sex modulates sensory

42 responses to pheromones and food [12, 19-23]. In Drosophila, chemoreceptor expression 
43 differs by sex [24-26] and chemosensory processing is modulated by female reproductive state

44 [27]. In mice, biological sex shapes sex differences in pheromone detection [28] and hormonal

45 signals of female reproductive status can act directly on sensory neurons to influence their

46 sensitivity to pheromonal and olfactory cues [29-31]. However, how biological sex and

47 reproductive state intersect with feeding status and other dimensions of internal state to

48 adaptively modulate behavior is largely unexplored.

50 investigating the mechanisms by which multiple internal and external cues are integrated to

51 balance drives and influence behavioral choice [32]. Under most circumstances, individual

52 worms are efficiently retained by a small spot of bacterial food. However, well-fed adult males

53 tend to leave a food source long before it is depleted, abandoning it in search of a suitable

54 mate [23]. Multiple mechanisms are important for the prioritization and execution of this

55 exploratory behavior, including input from male-specific sensory neurons that may tonically

56 signal the absence of potential mates [33] and peptidergic signaling through the PDFR-1

57 receptor [34, 35]. Additionally, adult males diminish their attraction to some food-related cues

58 compared to adult hermaphrodites and larval males, thereby promoting exploration over

59 feeding $[12,21]$. This occurs in part through repression of odr-10, the chemoreceptor for the

60 food-associated odorant diacetyl $[12,36]$. Downregulated odr-10 expression in adult males is

61 influenced by the genetic sex of the AWA neurons, the chemosensory neuron pair that

62 expresses this receptor [12], as well as a temporal cue signaling the transition to adulthood,

63 controlled by the heterochronic genes lep-2 and lep-5 [37]. 
65 the drive to mate with the drive to feed. If males are deprived of food for several hours and then placed on a food source, food-leaving behavior is transiently suppressed, returning to

67 baseline levels only after several hours of re-feeding [23]. Food-deprivation transiently activates odr-10 expression in males and increases odr-10-dependent food attraction, indicating that sensory plasticity is a component of this behavioral switch [12].

71 sensory behavior, we investigated the mechanisms that activate odr-10 expression upon food

72 deprivation in C. elegans adult males. In doing so, we identified a series of regulatory

73 interactions that couple feeding to low odr-10 expression, together constituting a neuroendocrine feedback loop involving the TGF 3 -superfamily signal DAF-7, the Insulin-IGF-1like signaling (IIS) receptor DAF-2, and its primary effector, the FoxO transcription factor DAFmechanism is largely male-specific, likely as a consequence of the recently described malespecific expression of daf-7 in the ASJ neurons [38]. odr-10 expression is also increased in response to food-deprivation in hermaphrodites, but this increase is primarily mediated by distinct mechanisms. Surprisingly, the signal that initiates this loop in males is not an internal

81 metabolic cue: starvation has little effect on odr-10 if it occurs in the presence of food stimuli,

82 but blocking the perception of food cues is sufficient to activate odr-10 expression even in well-

83 fed males. Together, these findings identify a sex-specific neuroendocrine feedback mechanism that couples sensory perception to chemoreceptor expression, thereby contributing to the 85 homeostatic balance of behavioral drives. 
RESULTS we examined odr-10 expression in males carrying mutations in regulatory pathways previously associated with feeding state. We first considered the insulin/IGF-1 signaling (IIS) pathway, since the activity of $d a f-2$, the receptor for the $C$. elegans IIS pathway, promotes food-leaving behavior in well-fed males [23]. Using the translational ODR-10::GFP fosmid reporter fsEx295 [12], we found that well-fed daf-2 mutant males exhibited high odr-10 expression, comparable to that seen in food-deprived wild-type males (Fig. 1C). This upregulation was eliminated in daf-

97 16; daf-2 double mutants, indicating that the activation of odr-10 by reduced insulin signaling depends on daf-16, the FoxO transcription factor that is the canonical effector of IIS in $C$. diacetyl, the ligand for odr-10 (Fig. 1D).

102 change in IIS, we examined ODR-10::GFP expression in food-deprived daf-16 males. Unlike wild-

103 type, daf-16 males exhibited no change in reporter expression upon food deprivation (Fig. 1E).

104 Similarly, starvation elicited a large increase in diacetyl attraction in wild-type males but had

105 only a very minor effect in daf-16 mutants (Fig. 1F). Together, these findings indicate that food 106 deprivation upregulates odr-10 through decreased IIS and increased daf-16 activity. However, 
107 they do not rule out the possibility that daf-16 is simply a permissive activator, necessary for

108 odr-10 expression under all circumstances. To test this, we examined larval males. As shown

109 previously, L3 males exhibit high odr-10 expression, comparable to that of L3 hermaphrodites,

110 with low odr-10 expression in males emerging only upon the transition to adulthood $[12,37]$.

111 We found that larval odr-10 expression was largely unaffected in daf-16 mutant males (Fig.

$112 \mathrm{~S} 1 \mathrm{~A})$, ruling out a general requirement of $d a f-16$ for all odr-10 expression. This indicates that an

113 increase in daf-16 activity likely underlies the activation of odr-10 in food-deprived adult males

114 (Fig. 11).

115

The response of odr-10 to food deprivation differs by sex

Our previous work indicated that the induction of odr-10 expression by food deprivation

118 occurred only in males [12]. However, by qRT-PCR, we observed a significant increase in odr-10

119 mRNA upon food deprivation in hermaphrodites (Fig. S1B). Furthermore, a recent report found

120 that $o d r-10$ expression was increased in daf-2 mutant hermaphrodites [39]. Therefore, we

121 considered the possibility that kyls53, the ODR-10::GFP reporter used previously, might be

122 prone to ceiling effects that could prevent the detection of increased fluorescence in food-

123 deprived hermaphrodites. When we examined hermaphrodites carrying the ODR-10::GFP

124 fosmid reporter $f s E x 295$, whose baseline expression level is lower than that of $k y / s 53$, we found

125 that food deprivation did indeed elicit a significant increase in GFP intensity (Fig. 1B). Thus, odr-

12610 expression is sensitive to food availability in both sexes, providing a homeostatic mechanism

127 by which the absence of food could increase in an animal's sensitivity to food odor. 
Despite this qualitatively similar response to food deprivation, however, we observed

129 sex differences in the underlying mechanisms. Unlike males, daf-2 mutant hermaphrodites

130 exhibited no apparent change in ODR-10::GFP intensity (Fig. 1G). Consistent with this, we found

131 that daf-16 was not required for the starvation-induced increase of odr-10 expression in

132 hermaphrodites (Fig. 1H). The apparent discrepancy between our findings and the qRT-PCR

133 results of Hahm et al. [39] might indicate an important role for post-transcriptional regulation

134 of odr-10 in hermaphrodites; further studies are needed to evaluate this and other possibilities.

135 Nevertheless, these findings demonstrate that IIS is not the primary mediator of odr-10

136 activation by food deprivation in hermaphrodites and that the sexes differ in the nature of the

137 mechanisms that link feeding state to odr-10 expression.

odr-10 is likely a direct target of daf-16

141 autonomously. We found that expression of daf-16f cDNA under the control of the AWA-

142 expressed promoter Pgpa-4 6 [12] was sufficient to restore odr-10 expression in starved daf-16

143 males (Fig. 2A). Consistent with this, we detected DAF-16::GFP in AWA nuclei in daf-2 mutant

144 males (Fig. 2B). In contrast, intestine-specific expression of daf-16 had no effect on odr-10 (Fig.

145 S2A, B).

147 DAF-16. Supporting this, a putative DAF-16 binding element was recently identified $809 \mathrm{bp}$

148 upstream of the odr-10 start codon [39]. To ask whether DAF-16 might act through this site, we 
generated two Podr-10::GFP reporters, one containing roughly $1 \mathrm{~Kb}$ of the wild-type odr-10 promoter (Podr-10 $\left.{ }^{\mathrm{WT}}\right)$ and one in which this element was disrupted by changing three conserved nucleotides (Podr-10 $0^{\Delta \text { DAF-16) }}$ (Fig. 2C). We observed a significant induction of Podr$10^{\mathrm{WT}}::$ GFP expression upon food deprivation, but detected no change in Podr-10 ${ }^{\triangle \mathrm{DAF}-16}:: \mathrm{GFP}$

(Fig. 2D, E). These results show that the induction of odr-10 by food deprivation in males is mediated at least in part at the transcriptional level. Moreover, they show that DAF-16 functions in AWA and that odr-10 is likely to be a direct target of the $C$. elegans IIS pathway.

\section{TGFB signaling acts upstream of IIS to regulate odr-10 in males}

We also considered a role for the BMP/TGF $\beta$-family factor DAF-7 in regulating odr-10. In larval hermaphrodites [40-42], daf-7 expression in the ASI neurons decreases in response to nutritional stress. Previous work has shown that this signal is involved in many food-related aspects of $C$. elegans development and physiology, including entry into the dauer stage, fertility, fat metabolism, satiety quiescence, immune response, and lifespan [40-53]. daf-7 also regulates the expression of several chemoreceptor genes in adult hermaphrodites, though odr10 was not found to be among these [54]. Interestingly, a recent study found that daf-7 is malespecifically expressed in the ASJ neurons, where it promotes food-leaving behavior [38].

We found that well-fed daf-7 mutant males displayed significant upregulation of ODR10::GFP (Fig. 3A), indicating that this signal also has a role in repressing odr-10 expression. This regulation appears to be male-specific, as we detected no change in ODR-10::GFP in daf-7 mutant hermaphrodites (Fig. 3A). To further explore this, we genetically masculinized the 
nervous system of hermaphrodites by pan-neural expression of the male sex-determination

171 regulator fem-3 [19, 21]. Consistent with previous work [12], this was sufficient to reduce

172 expression of ODR-10::GFP to male-typical levels (Fig. 3B). Further, pan-neural masculinization

173 made hermaphrodite odr-10 expression sensitive to $d a f-7$ loss, with odr-10 levels increasing to

174 an extent comparable to that seen in wild-type males (Figs. 3A, B). Thus, sex differences in the

175 nervous system itself account for the male-specificity of the regulation of odr-10 by daf-7. As

176 pan-neural masculinization is sufficient to activate daf-7 expression in ASJ in hermaphrodites

177 [38], its expression in this neuron likely explains the sex-specificity of its role in regulating odr-

17810.

Next, we explored the relationship between IIS and daf-7 signaling. Crosstalk between

180 these pathways has been found previously: several insulin signaling peptide genes are

181 transcriptionally regulated by daf-7 signaling $[55,56]$ and daf-7 mutants show increased nuclear

182 localization of the IIS effector DAF-16 [53, 57]. Consistent with this, we found that daf-16 was

183 required for the strong induction of odr-10 in daf-7 mutant males, such that ODR-10::GFP

184 intensity was only slightly higher in daf-16; daf-7 double mutants than in daf-16 single mutants

185 (Fig. 3C). This suggests that daf-7 primarily regulates odr-10 expression by modulating IIS, with 186 secondary effects through other mechanisms (Fig. 3E).

To better understand the regulation of $o d r-10$ by daf-7, we sought to determine the

188 cellular focus of TGF $\beta$ pathway activity. First, we found that males carrying a mutation in daf-8,

189 a SMAD downstream of daf-7 [58], phenocopied the high odr-10 expression of daf-7 mutants

190 (Fig. 3D). Further, expression of a daf-8 cDNA under the control of its own promoter or a pan-

191 neural promoter was sufficient to reduce odr-10 expression in daf-8 mutant males (Fig. 3D). 
However, we detected no rescue when we expressed daf-8 specifically in AWA (Fig. 3D). state regulation of $o d r-10$ in males.

\section{Food perception, rather than internal metabolic state, regulates odr-10 in males}

During food deprivation, animals experience both metabolic stress and the loss of

209 deprived entirely of bacterial food (Fig. S3). Despite this metabolic stress, however, these

210 animals displayed only a very modest increase in ODR-10::GFP expression (Fig. 4A). Next, we re-

211 exposed food-deprived animals to either control or aztreonam-treated bacteria for $24 \mathrm{~h}$. In

212 both cases, ODR-10::GFP expression returned to baseline levels, even though those cultured on 
213 aztreonam-treated bacteria presumably remained in a starved state (Fig. 4B). Thus, while

214 metabolic stress may play a small role in odr-10 activation upon food deprivation in adult

215 males, the loss of chemosensory cues appears to be the primary driver of this regulation.

To ask whether this principle applies in both sexes, we exposed adult hermaphrodites to

217 aztreonam-treated bacteria. Unlike males, hermaphrodites responded with full induction of

218 ODR-10::GFP, mounting a response indistinguishable from that elicited by food deprivation (Fig.

$2194 \mathrm{4C}$ ). This indicates that the role of food signals in the regulation of odr-10 differs markedly by

220 sex, such that the induction of odr-10 in food-deprived hermaphrodites appears to result

221 primarily from nutritional stress, rather than the loss of chemosensory cues.

This apparent sex-specific sensitivity to nutritional status suggested that reducing food

223 quality or intake might increase odr-10 expression in hermaphrodites but not in males. To test

224 this, we grew animals on a low-quality food source, heat-killed E. coli, for 16-18 $\mathrm{h}$. Under these

225 conditions, odr-10 expression in males was comparable to that observed in males grown on

226 control food, but expression in hermaphrodites was significantly increased (Figs. 4D, E). We also

227 examined the effects of reducing food intake by assaying ODR-10::GFP in eat-2 mutants, in

228 which the pharyngeal pumping rate and the intake of food are reduced [60]. We found that this

229 had no detectable effect on odr-10 expression in males, but that odr-10 expression in eat-2

230 hermaphrodites was significantly higher than in control animals (Fig. 4F). These findings

231 reinforce the idea that $o d r-10$ is regulated primarily by food-derived sensory signals in males

232 and by internal metabolic state in hermaphrodites. 

signals on odr-10 expression in males might be mediated by daf-7 signaling, we examined Pdaf7::GFP expression in animals cultured on aztreonam-treated $E$. coli. In control experiments, we verified that the male-specific expression of Pdaf-7::GFP in ASJ was dramatically reduced upon

240 food deprivation, but that expression in ASI was relatively unchanged (Fig. 4G, H). In contrast,

241 culturing animals on inedible food did not bring about downregulation of daf-7 expression in

242 ASJ or in ASI (Fig. 4G); on the contrary, for unknown reasons, daf-7 expression in ASJ increased

243 when males were grown on inedible food (Fig. 4G). This suggests that, in males, daf-7 levels in

244 ASJ are an internal representation of the detection of food rather than the level of physiological

245 satiety. Moreover, these findings indicate that a reduction of daf-7 expression couples

246 decreased food perception to the activation of odr-10 in adult C. elegans males.

248 function should alter odr-10 expression. To test this, we examined animals carrying mutations

249 in tax-2 and tax-4, genes that encode subunits of a cyclic nucleotide-gated cation channel

250 required for the function of a subset of $C$. elegans chemosensory neurons [61, 62]. We found

251 significant upregulation of $o d r-10$ in well-fed tax-2 and tax-4 mutant males (Fig. 5A), supporting

252 this hypothesis. Notably, tax-2 and tax-4 are not expressed in AWA and are not required for

253 AWA-mediated chemosensory behavior [63], indicating that sensory perception regulates odr-

25410 non-cell-autonomously. In hermaphrodites, loss of tax-2 caused a modest increase in odr-10

255 expression, while loss of tax-4 resulted in a trend toward increased expression (Fig. 5B). Thus, 
while chemosensory signals are not the primary modulator of odr-10 expression in

257 hermaphrodites, they likely contribute to its regulation.

259 IIS, the elevated odr-10 expression in tax-4 mutant males should depend on daf-16. Indeed, we

260 found that the odr-10 expression phenotype of tax-4 males was strongly suppressed in daf-16;

261 tax-4 double mutants (Fig. 5A). Similarly, daf-16 was essential in hermaphrodites for the

262 modest effect of tax-4 loss on odr-10 expression (Fig. 5B). Thus, the sensory signals mediated by

263 tax-4-positive neurons likely act through IIS to repress odr-10 expression. Interestingly, previous

264 work has shown that the expression of $d a f-7$ is reduced in tax-4 mutant hermaphrodites [45],

265 suggesting that the increase in odr-10 expression we observed in tax-4 males could result from

266 a reduction in daf-7 expression in ASJ and/or ASI. Consistent with this, we found that Pdaf-

267 7::GFP expression in tax-4 males was strongly reduced in ASI and was virtually undetectable in

268 ASJ (Fig. 5C, E, F). Thus, the high expression of odr-10 in tax-4 males likely results from a

269 reduction in daf-7 expression. Together, this suggests that removal from food decreases the

270 activity of a subset of tax-4 neurons, leading to a reduction in daf-7 production in ASJ, reduced

271 IIS pathway activity, and increased odr-10 expression.

273 elegans sensory neurons, including ASI and ASJ [61, 62]. To ask whether tax-4 acts cell-

274 autonomously in these neurons to promote the expression of daf-7, we carried out cell-type-

275 specific rescue experiments. Although rescue of tax-4 under the endogenous Ptax-4 promoter

276 reduced the elevated $o d r-10$ expression of tax-4 mutant males, we observed no such effect

277 when tax-4 was expressed specifically in ASJ, ASI, or both neurons simultaneously (Figs. 5D and 
S4A). Consistent with this, ASI/ASJ-specific expression of tax-4 did not restore daf-7 expression in tax-4 mutants (Fig. 5E, F). These results suggest that sensory perception regulates daf-7 noncell-autonomously, and that chemosensation in ASI and ASJ does not regulate odr-10.

To narrow the focus of sensory function required for $d a f-7$ and odr-10 expression, we used tax-2(p694), an unusual allele that limits tax-2 function to ASI, ASJ, and four other pairs of neurons: AWB, AWC, ASK, and ASG [61, 64]. Unlike tax-2 null mutants, tax-2(p694) males showed no increase in odr-10 expression (Fig. 5A), indicating that one or more of this group of neurons is sufficient to transduce the sensory signals that repress odr-10 in males on food. Examining these classes individually, we found that ceh-36 mutant males, in which AWC does not properly differentiate, displayed no changes in odr-10 expression (Fig. S5A). Similarly, odr10 was unaltered in animals in which AWB or ASK was genetically ablated (Fig. S5B, C). Furthermore, we found that ASG-specific expression of tax-4 failed to reduce odr-10 expression in tax-4 males (Fig. S5D). Thus, we conclude that, in well-fed males, odr-10 expression is repressed by the distributed detection of food-derived chemosensory cues (Fig. 5G).

\section{tax-2/tax-4-dependent sensory signals, acting through IIS, contribute to the balance between}

\section{feeding and exploration}

Previously, we showed that overexpression of odr-10 in adult males was sufficient to reduce food-leaving, while loss of odr-10 in adult hermaphrodites increased it [12]. The size of these effects is relatively small, consistent with the idea that modulation of odr-10 is just one of several mechanisms regulating this behavior [32]. Therefore, we sought to assess the 
contribution of the feedback loop we identify here to the feeding-vs.-exploration decision. To do so, we measured food-leaving behavior in wild-type, tax-4, and daf-16; tax-4 males. tax-4

301 mutant males should mimic a state of prolonged food absence, with low daf-7 and high odr-10 expression inhibiting food-leaving behavior $[12,38]$. However, because TAX-2/TAX-4 neurons are themselves important for food detection [65], loss of tax-4 function seems likely to increase

304 food-leaving, making it difficult to predict how the behavior of these animals might differ from wild-type. We found that tax-4 mutant males exhibited modestly reduced food-leaving

306 compared to WT males (Fig. 5G), suggesting that the strong food-leaving defect expected to

307 result from low daf-7 expression [38] is counteracted by the absence of food detection by TAX$308 \quad$ 2/TAX-4 neurons.

Because odr-10 activation in tax-4 mutants requires daf-16, the loss of daf-16 might be

310 expected to increase the food-leaving behavior of tax-4 males. Indeed, at $24 \mathrm{hr}$, daf-16; tax-4

311 males exhibited slightly more food-leaving behavior than did tax-4 males (Fig. 5G). Because

312 constitutive loss of tax-4 and daf-16 likely causes broad changes in worm behavior and

313 physiology, these results should be interpreted cautiously. Nevertheless, they are consistent

314 with the idea that the sensory- and daf-16-dependent modulation of odr-10 contributes to 315 recalibrating the feeding-vs.-exploration balance in food-deprived males.

\section{DISCUSSION}

Homeostatic regulation is a central feature of many animal behaviors and drives,

319 including feeding, sleep, and reproduction, and is often driven by internal signals representing 
aspects of physiological and metabolic state [66]. In C. elegans males, odr-10, the

321 chemoreceptor for the food-associated odor diacetyl, helps tune the behavioral equilibrium

322 between mate-searching and feeding. In well-fed adults, low expression of odr-10 reduces food

323 attraction and promotes food-leaving; food deprivation upregulates odr-10, contributing to

324 increased food attraction and decreased food-leaving [12]. Here, we identify a neuroendocrine

325 feedback mechanism that regulates odr-10 expression as a function of feeding state.

326 Surprisingly, the low odr-10 expression typical of well-fed adult males is not enabled by signals

327 that reflect nutritional status. Rather, the presence of food itself engages a neuroendocrine

328 feedback mechanism that downregulates odr-10, blunts chemosensory acuity, and helps

329 balance the nutritional and reproductive needs of the adult $C$. elegans male.

In the model we propose (Fig. 6), multiple food-derived chemosensory cues are

331 detected by a subset of sensory neurons that express a cyclic-nucleotide-gated channel

332 comprising the subunits TAX-2 and TAX-4. Our results suggest that multiple TAX-2/TAX-4-

333 positive neurons contribute at this step. Using an unusual allele of tax-2, we find that the

334 activity of six pairs of chemosensory neurons (AWB, AWC, ASG, ASI, ASJ, and ASK) is sufficient

335 to provide the "on-food" signal. Rescue and ablation experiments indicates that this signal is

336 distributed among multiple members of this group, at least four of which are known to detect

337 food-associated signals [65]. Because bacterial food sources emit complex and variable

338 chemical signatures [67], it is not surprising that adult males use multiple streams of

339 information to assess the presence of food.

Next, these sensory signals converge to regulate expression of the TGF $\beta$-superfamily

341 ligand DAF-7. Production of this signal by the ASI neurons has been repeatedly shown to 
represent favorable environmental conditions, both in larvae and adults, and daf-7 expression

343 is known to be regulated by sensory signals in hermaphrodites [40-45, 48, 49, 51, 68].

344 Interestingly, recent work has identified a sex difference in daf-7 expression, such that well-fed

345 adult males also produce DAF-7 in the ASJ neurons, where its expression promotes food-leaving

346 behavior [38]. Our results indicate that daf-7 expression in ASJ responds to sensory information

347 from TAX-2/TAX-4 neurons: both in the absence of food and in tax-4 mutants, daf-7 expression

348 in ASJ is markedly reduced. Interestingly, although both ASJ and ASI are TAX-2/TAX-4 neurons,

349 we find that the sensory function of these cells alone is insufficient to activate daf-7 expression

350 in the presence of food. Thus, non-cell-autonomous signals from AWB, AWC, ASK, and/or ASG

351 are transmitted to ASJ and ASI, indicating that these neurons integrate multiple dimensions of

352 sensory information into a single environmental-state quality signal.

daf-7 is known to influence many aspects of $C$. elegans development, physiology, and

354 behavior, including decisions about whether to remain on a food source [38, 47]. Many of these

355 effects are mediated by regulation of the RIC and RIM interneurons via the SMAD daf- 8 and

356 other members of the canonical TGF $\beta$ signaling pathway $[42,58]$. However, our results indicate

357 that, with respect to the regulation of $o d r-10$ in adult males, daf-8 does not act in RIC/RIM, nor

358 in AWA, the neuron in which odr-10 is expressed. However, because the odr-10 expression

359 defect of daf-8 males can be rescued by pan-neural expression of $d a f-8$, the $d a f-7$ signal must

360 act somewhere in the nervous system.

362 maintaining low odr-10 expression in well-fed adult males. Males bearing mutations in the IIS

363 receptor daf-2 exhibit high odr-10 expression even in the well-fed state; this phenotype is 
suppressed by loss of the FoxO family factor daf-16, the primary effector of $C$. elegans IIS [69].

365 Importantly, loss of daf-16 prevents the upregulation of odr-10 upon food deprivation,

366 indicating that, in wild-type adult males, removal from food leads to downregulation of daf-2

367 activity and the activation of daf-16. Furthermore, the high odr-10 expression seen in daf-7 and

368 in tax-4 mutant males largely depends on daf-16 function, strongly suggesting that these

369 components of the feedback mechanism act upstream of IIS. Though daf-7 and IIS pathways act

370 largely independently in the dauer development decision [70-72], daf-7 can also act upstream

371 of IIS [53, 55-57]. Thus, we favor a model in which daf-8 acts in the nervous system to regulate

372 the production and/or release of one or more insulin-like peptides (ILPs). Because the $C$.

373 elegans genome encodes 40 ILPs [69], further studies are needed to identify the specific ligands

374 involved in this mechanism and the site of their production.

In the last steps of the feedback mechanism, one or more ILPs act directly on the AWA

376 chemosensory neurons. Cell-type-specific rescue experiments demonstrate that daf-16 acts in

377 AWA; moreover, we find that odr-10 is a very likely to be direct transcriptional target of daf-16.

378 While daf-16 is best known for its role in activating regulators of stress response and

379 reprogramming metabolism, our results, together with those of others $[5,7,73]$ show that IIS

380 can also directly regulate chemosensory function. This increase in odr-10 expression, likely just

381 one part of a suite of changes in AWA physiology, increases sensitivity to diacetyl and to

382 bacterial food. Interestingly, AWA has recently been found to play a male-specific role in

383 detecting volatile (non-ascaroside) pheromones released by hermaphrodites [74]. One

384 intriguing question for future work will be to ask whether the mechanism we describe here also

385 regulates the pheromone-detection function of AWA, perhaps blunting it in food-deprived 
387 precedent in $C$. elegans $[7,54,75]$; our work shows that this mechanism is deployed by $C$.

388 elegans as a component of sex- and food-dependent behavioral plasticity.

While reproductive drives are well appreciated as key motivators of animal behavior,

390 the mechanisms underlying sex differences in such drives remain largely mysterious. Biological

391 sex can also influence other drives and their relative prioritization-for example, sleep

392 homeostasis in Drosophila is subject to regulation by male-specific sexual arousal [76, 77] - but

393 the mechanistic interactions between sex and other internal states has received little attention.

394 We find that the chemosensory feedback loop described here is largely sex-specific. While food

395 deprivation boosts odr-10 expression in both sexes, only in males is this primarily driven by

396 sensory feedback, daf-7, and the IIS-mediated activation of odr-10. In hermaphrodites, these

397 mechanisms have relatively little contribution to odr-10 regulation. Instead, a physiological cue,

398 mediated by unknown signals, is the primary influence on odr-10 in food-deprived

399 hermaphrodites. The basis for this sex difference lies within the nervous system itself, as pan-

400 neural masculinization of the hermaphrodite nervous system is sufficient to confer sensitivity to

401 daf-7 on odr-10 expression. The recent finding of male-specific expression of daf-7 in ASJ likely

402 underlies the male-specificity of the mechanism we describe here, as the expression of daf-7 in

403 ASJ is particularly sensitive to food [38] and to sensory signals (this work) compared to that in

404 ASJ. Furthermore, pan-neural masculinization (but not masculinization of ASJ alone) activated

405 daf-7 expression in ASJ in hermaphrodites [38]. Together with our finding that multiple TAX-

406 2/TAX-4-family chemosensory neurons are important for $d a f-7$ and odr-10 regulation in males, 
407 this suggests that male-specific properties of multiple sensory neurons might alter the

408 regulatory logic by which sensory signals are integrated by daf-7.

Why might males and hermaphrodites use different mechanisms to couple feeding

410 status to odr-10 expression? Differences in the ways that the sexes invest resources to optimize

411 reproductive fitness may offer a clue. The production of sperm is relatively cheap; males may

412 instead allocate resources to generating higher levels of motor activity in the service of mate-

413 searching $[78,79]$. In contrast, the production of oocytes is resource-intensive, requiring the

414 continuous synthesis of many macromolecular components. As such, it is possible that relying

415 on direct measurements of food consumption and physiological state provides hermaphrodites

416 with a more reliable indicator of the pressure to find new sources of nutrition. Numerous

417 signaling mechanisms -including daf-7 signaling; IIS; metabotropic signaling by glutamate,

418 monoamines, and neuropeptides; the kinase AMPK; and RICTOR/TORC2 signaling - have been

419 implicated in linking feeding and nutritional uptake to metabolism and behavior in

420 hermaphrodites, e.g. [42, 43, 46-48, 80-96]. Other than daf-7 and IIS, one or more of these

421 "gut-to-brain" signals seem likely candidates for regulating odr-10 in hermaphrodites. Further,

422 the functional significance of increased odr-10 expression in a food-deprived hermaphrodite is

423 unclear, as hermaphrodites are already strongly attracted to food, even in the well-fed state.

424 Perhaps higher odr-10 expression makes food detection even more efficient or helps override

425 inhibitory signals that prevent the consumption of lower-quality food in well-fed animals.

427 would seemingly allow rapid assessment of environmental state, but behavioral plasticity in

428 food-leaving behavior and odr-10 expression occurs over hours, not seconds or minutes. It may 
429 be that the integration of multiple chemosensory streams by daf-7 occurs over a longer time

430 scale, generating a time-averaged signal that reflects recently encountered environmental

431 conditions. Further, the time needed for signaling downstream of daf-7 also likely contributes

432 to the delay between removal from food and the increase in ODR-10 abundance. Another issue

433 worth considering is that males also use chemosensory information about the presence of food

434 when deciding whether to copulate with a hermaphrodite. In the absence of food, males mate

435 poorly with hermaphrodites, possibly to avoid investing in progeny that will be born into an

436 unfavorable environment [97]. Interestingly, inedible food provides an environment nearly as

437 permissive for mating success as does control food, indicating that males use chemosensory

438 cues about food availability when making this decision [98]. The relationship of these cues to

439 those that regulate odr-10 are unknown, but it seems likely that they are related.

The importance of chemosensory cues in calibrating odr-10 expression and, indirectly,

441 the feeding-vs.-exploration balance, contributes to a growing appreciation of the importance of

442 chemosensory signals in regulating behavior, metabolism, and internal state in C. elegans.

443 Chemosensory cues have long been known to be important for the developmental decision to

444 enter the dauer state, a long-lived, stress-resistant larval stage whose entry can be triggered by

445 the absence of food and exposure to population-density pheromones, and the daf-7 TGF $\beta$ and

446 daf-2 IIS pathways are the primary regulators of this decision [72]. In adult hermaphrodites,

447 chemosensory cues themselves have been shown or suggested to alter gene expression,

448 physiology, behavior, and lifespan e.g. [7, 42, 44, 46, 48, 68, 89, 99-102]. By establishing an

449 important role for such signals in the male-specific regulation of chemosensory function, our 
work shows that chemosensory signals contribute to the balance between feeding and mating

451 drives, and that they do so at least in part by influencing chemosensory function itself.

452

453

454 ACKNOWLEDGMENTS

455

For discussion and critical feedback, we thank current and past members of the Portman lab and

456 the Western New York Worm Group. We are particularly grateful to Deborah Ryan, who contributed the

457 data shown in Figure S1B. Some strains used in this work were provided by the Caenorhabditis Genetics

458 Center, which is funded by NIH Office of Research Infrastructure Programs (P40 OD010440). This work

459 was supported by grants from the NIH (R01 GM108885 and R01 GM130136) to D.S.P.

460

$461 \quad$ AUTHOR CONTRIBUTIONS

462

L.W. and D.P. designed the experiments; L.W. and R.M. conducted the experiments; L.W., R.M.,

463 and D.P. analyzed and interpreted the results; and L.W. and D.P. wrote the manuscript.

464

465 EXPERIMENTAL PROCEDURES

466 Strains

Strains were grown on E. coli OP50 using standard methods [103] and maintained at $20^{\circ} \mathrm{C}$.

468 Unless otherwise stated, all strains contained him-5(e1490) in order to obtain a high number of males;

469 this is considered the wild-type for the purposes of these studies. Strains carrying mutations in daf-2,

470 daf-7, or daf-8 were maintained at $15^{\circ} \mathrm{C}$ to prevent dauer entry. In these cases, all paired control strains 
471 were also maintained at $15^{\circ} \mathrm{C}$. Animals were transferred to $25^{\circ} \mathrm{C}$ (for daf-2 strains) or $20^{\circ} \mathrm{C}$ (for daf-7 and

472 daf-8 strains) as L4 larvae; scoring of one-day adults was carried out the following day. See Table S1 for a

473 complete list of $C$. elegans strains used in this work.

475 Quantification of ODR-10::GFP, Podr-10::GFP, and Pdaf-7::GFP

All animals used for experiments were sex-segregated as L4 larvae the day before scoring. One-

477 day old adults were mounted on a $4 \%$ agarose pad and immobilized with levamisole. GFP fluorescence

478 was observed using a 63x PlanApo objective on a Zeiss Axioplan 2. Due to the complexity of the

479 structure of the AWA cilia, accurate computer-based quantification of ODR-10::GFP fluorescence

480 intensity was not possible. ODR-10::GFP intensity was therefore scored using a scale of 0-3 (0=absent,

481 1=faint, $2=$ =moderate, $3=$ bright) as previously described $[12,37]$. When possible, the experimenter was

482 blinded to genotype. For quantification of Podr-10::GFP and Pdaf-7::GFP expression, the integrated

483 density of GFP was calculated using FIJI [104]. Images were obtained under constant imaging conditions

484 with the cell of interest in center of the field. Background was calculated by taking the average mean

485 fluorescence of three random sections of background within the area of the animal. Total cell

486 fluorescence was calculated as (integrated density of GFP in the region of interest) - (mean background

487 fluorescence $x$ area of the region of interest). 
494 performed on a Bio-Rad MyiQ2 icycler with iQ SYBR Green Supermix (BioRad) using $2 \mu$ of cDNA with

495 reactions in triplicate. Cycling conditions were: 1 cycle of $95^{\circ} \mathrm{C}$ for $5 \mathrm{~min} ; 45 \mathrm{cycles}$ of $95^{\circ} \mathrm{C}$ for $30 \mathrm{~s}, 60^{\circ} \mathrm{C}$

496 for $30 \mathrm{~s}$, and $72^{\circ} \mathrm{C}$ for $45 \mathrm{~s}$, followed by melting curve analysis to verify product specificity. Standard

497 curves were generated for each primer using three serial dilutions of cDNA to determine an efficiency

498 estimate for each run. Threshold cycles (Ct) were determined by the Bio-Rad iQ5 optical system

499 software, which were used with efficiency estimates to calculate relative odr-10 gene expression by the

500 Pfaffl analysis method [105]. Two reference genes, $c d c-42$ and Y45F10D.4, were used for normalization

501 [106]. odr-10 expression in starved hermaphrodites is shown normalized to levels detected in well-fed

502 hermaphrodites.

504 Behavioral Assays

506 all cases, the investigator was blinded to genotype.

\section{Aztreonam-treated OP50}

$510 \mathrm{OP} 50$ was grown in $\mathrm{LB}$ medium at $37^{\circ} \mathrm{C}$ with shaking to log-phase growth. Aztreonam was then added to

511 a final concentration of $10 \mu \mathrm{g} / \mathrm{ml}$ and cultures were incubated for 3 additional hours without shaking.

512 Bacteria were then seeded onto freshly made (same-day) NGM plates containing aztreonam (10 $\mu \mathrm{g} / \mathrm{ml})$.

513 To avoid transferring untreated bacteria, worms were washed at least 3 times with M9 buffer before

514 being placed on aztreonam plates. Animals were scored after 16-18 h. For paired control experiments, 
515 starved animals were plated on unseeded NGM plates containing $10 \mathrm{ug} / \mathrm{ml}$ aztreonam to control for any

516 effects of aztreonam on gene expression.

518 Molecular biology and generation of transgenic strains

All cDNAs were amplified from total RNA extracted from him-5(e1490) cultures. (See Table S2

520 for primer sequences.) The resulting cDNAs were cloned into pDONR221 and recombined via Multisite

521 Gateway Cloning (Invitrogen). The Podr-10 ${ }^{\triangle \mathrm{DAF}-16}:$ :GFP transgene was generated by Gibson assembly

522 [108]. Plasmids were injected into animals at a concentration of 20-50 ng/ul of rescue construct and 100

$523 \mathrm{ng} / \mathrm{ul}$ of Pelt-2::GFP co-injection marker.

525 Starvation experiments

One-day old adults were washed at least three times with M9 buffer and then transferred to

527 NGM plates with either E. coli OP50 or no food. For GFP quantification experiments, animals were

528 scored after 16-18 h. For behavioral assays, animals were tested after $12 \mathrm{~h}$.

$530 \quad$ Heat killed E. coli OP50

531 Liquid cultures of E. coli OP50 were incubated at $75^{\circ} \mathrm{C}$ for $1 \mathrm{~h}$. Heat-killed bacteria were then

532 plated and used for experiments on the following day. 
For categorical data (e.g., fluorescence intensity of ODR-10::GFP and behavior in the food-

536 leaving assay), data were analyzed using non-parametric tests. For pairwise comparisons, Mann-

537 Whitney tests were carried out. For multiple comparisons, we used Kruskal-Wallis analysis with Dunn's

538 correction. For other analyses (e.g., of diacetyl chemotaxis and quantitated GFP fluorescence), data

539 were assumed to be normally distributed. In these cases, Welch's $t$ tests were used for pairwise

540 comparisons. For multiple comparisons, we used Brown-Forsyth and Welch one-way ANOVA tests with

541 Holm-Sidak's correction. In all experiments, only those comparisons necessary to test the hypotheses

542 under consideration were carried out. For this reason, graphs do not indicate the results of all possible

543 comparisons. Instead, all graphs show the results of all comparisons made. Dotted gray brackets indicate

544 comparisons in which the $p$ value was greater than 0.05 . In cases where $p$ was 0.05 or less, asterisks

545 indicate $p$ value ranges as follows: ${ }^{*} 0.01<p<0.05 ;{ }^{* *} 0.001<p<0.01 ;{ }^{* *} p<0.001$.

TABLE S1. Strain List.

\begin{tabular}{|l|l|}
\hline Strain & Genotype \\
\hline UR228 & daf-2(e1370) III; him-5(e1490) V \\
\hline UR460 & him-5(e1490) V; kyls53[Podr-10::odr-10::GFP] X \\
\hline UR773 & pha-1(e2123) III; him-5(e1490) V; fsEx295[ODR-10::GFP fosmid + pBx1] \\
\hline UR910 & $\begin{array}{l}\text { dpy-5(e61) daf-8(e1393) I; him-5; kyls53; fsEx476 [Pgpa-4d6::daf-8cDNA::SL2::mCherry::unc-54 3' UTR } \\
+ \text { Pelt-2::GFP line 1] }\end{array}$ \\
\hline UR915 & dpy-5(e61) daf-8(e1393) I; him-5(e1490) V; kyls53X \\
\hline UR926 & him-5(e1490) V \\
\hline UR1018 & him-5(e1490) V; kyls53 X; fsls15; daf-7(e1372) III \\
\hline UR1067 & daf-16(mgDf47) l; him-5(e1490) V \\
\hline UR1100 & him-5(e1490) V; fsEx513[1134bpPodr-10::GFP + Pmyo-3::mCherry line 1] \\
\hline UR1101 & him-5(e1490); fsEx514[1134bpPodr-10::GFP daf-16 site mutated + Pmyo-3::mCherry line 1] \\
\hline UR1116 & daf-16(mgDf47) l; pha-1(e2123) III; him-5(e1490) V; fsEx295 \\
\hline UR1136 & $\begin{array}{l}\text { daf-16(mgDf47) l; pha-1(e2123) III; him-5(e1490) V; fsEx295; fsEx532[Pgpa4del6::daf-16f } \\
\text { cDNA::SL2::mCherry::unc-54 3' UTR + Pelt-2::GFP line1] }\end{array}$ \\
\hline UR1137 & $\begin{array}{l}\text { daf-16(mgDf47) l; pha-1(e2123) III; him-5(e1490) V; fsEx295; fsEx533[Pgpa4del6::daf-16f } \\
\text { cDNA::SL2::mCherry::unc-54 3' UTR + Pelt-2::GFP line2] }\end{array}$ \\
\hline
\end{tabular}




\begin{tabular}{|c|c|}
\hline UR1138 & $\begin{array}{l}\text { daf-16(mgDf47) l; pha-1(e2123) III; him-5(e1490) V; fsEx295; fsEx534[Pnhx-2::daf-16f } \\
\text { cDNA::SL2::mCherry::unc-54 3' UTR + Pelt-2::GFP line1] }\end{array}$ \\
\hline UR1139 & $\begin{array}{l}\text { daf-16(mgDf47) l; pha-1(e2123) III; him-5(e1490) V; fsEx295; fsEx535[Pnhx-2::daf-16f } \\
\text { cDNA::SL2::mCherry::unc-54 3' UTR + Pelt-2::GFP line2] }\end{array}$ \\
\hline UR1194 & $\operatorname{tax}-4(o k 3771)$ III; him-5(e1490) V; kyls53X \\
\hline UR1196 & tax-4(ok3771) III; him-5(e1490) V; ks/s2 \\
\hline UR1201 & tax-4(ok3771) III; him-5(e1490) V; fsEx295 \\
\hline UR1202 & daf-16(mgDf47) I; tax-4(ok3771) III; him-5(e1490) V; fsEx295 \\
\hline UR1203 & daf-2(e1370) III; him-5(e1490) V; fsEx295 \\
\hline UR1204 & daf-16(mgDf47) I; daf-2(e1370) III; him-5(e1490) V; fsEx295 \\
\hline UR1205 & daf-7(e1372) III; him-5(e1490) V; fsEx295 \\
\hline UR1206 & daf-7(e1372) III; daf-16(mgDf47) I; him-5(e1490) V; fsEx295 \\
\hline UR1222 & $\begin{array}{l}\text { tax-4(ok3771) III; him-5(e1490) V; kyls53 X; fsEx550[Ptax-4::tax-4c::SL2::mCherry::unc-54 3' UTR + } \\
\text { Pelt-2::GFP] }\end{array}$ \\
\hline UR1234 & $\begin{array}{l}\text { tax-4(ok3771) III; him-5(e1490) V; kyls53 X; fsEx553[Pgpa-4::tax-4c::SL2::mCherry::unc-54 3' UTR + } \\
\text { Pelt-2::GFP line 1] }\end{array}$ \\
\hline UR1235 & $\begin{array}{l}\text { tax-4(ok3771) III; him-5(e1490) V; ky/s53 X; fsEx554[Pgpa-4::tax-4c::SL2::mCherry::unc-54 3' UTR + } \\
\text { Pelt-2::GFP line 2] }\end{array}$ \\
\hline UR1236 & $\begin{array}{l}\text { tax-4(ok3771) III; him-5(e1490) V; kyls53 X; fsEx555[Pssu-1::tax-4c:::SL2::mCherry::unc-54 3' UTR + } \\
\text { Pelt-2::GFP line 1] }\end{array}$ \\
\hline UR1237 & $\begin{array}{l}\text { tax-4(ok3771) III; him-5(e1490) V; kyls53 X; fsEx556[Pssu-1::tax-4c::SL2:::mCherry::unc-54 3' UTR + } \\
\text { Pelt-2::GFP line 2] }\end{array}$ \\
\hline UR1238 & $\begin{array}{l}\text { tax-4(ok3771) III; him-5(e1490) V; kyls53 X; fsEx557[Pgpa-4::tax-4c::SL2:::mCherry::unc-54 3' UTR + } \\
\text { Pssu-1::tax-4c::SL2::mCherry::unc-54 3' UTR + Pelt2::GFP line 1] }\end{array}$ \\
\hline UR1239 & $\begin{array}{l}\text { tax-4(ok3771) III; him-5(e1490) V; kyls53 X; fsEx5588[Pgpa-4::tax-4c:::SL2:::mCherry::unc-54 3' UTR + } \\
\text { Pssu-1::tax-4c::SL2::mCherry::unc-54 3' UTR + Pelt2::GFP line 2] }\end{array}$ \\
\hline UR1284 & him-5(e1490); fsEx571[1134bpPodr-10::GFP daf-16 site mutated + Pmyo-3::mCherry line 2] \\
\hline UR1285 & him-5(e1490) V; ksIs2[Pdaf-7::GFP+rol-6(su1006)] \\
\hline UR1286 & $\begin{array}{l}\text { dpy-5(e61) daf-8(e1393) l; him-5; kyls53; fsEx572[Pdaf-8::daf-8cDNA::SL2::mCherry::unc-54 3' UTR + } \\
\text { Pelt-2::GFP line 1] }\end{array}$ \\
\hline UR1287 & $\begin{array}{l}\text { dpy-5(e61) daf-8(e1393) I; him-5; kyls53; fsEx573[Prab-3::daf-8cDNA::SL2::mCherry::unc-54 3' UTR + } \\
\text { Pelt-2::GFP line 1] }\end{array}$ \\
\hline UR1288 & $\begin{array}{l}\text { dpy-5(e61) daf-8(e1393) I; him-5; kyls53; fsEx574[Pgpa-4d6::daf-8cDNA::SL2::mCherry::unc-54 3' UTR } \\
+ \text { Pelt-2::GFP line 2] }\end{array}$ \\
\hline UR1289 & $\begin{array}{l}\text { dpy-5(e61) daf-8(e1393) I; him-5; kyls53; fsEx575[Ptdc-1::daf-8cDNA::SL2::mCherry::unc-54 3' UTR + } \\
\text { Pelt-2::GFP] }\end{array}$ \\
\hline UR1290 & $\begin{array}{l}\text { daf-2(e1370) III; him-5(e1490); zIs356[daf-16p::daf-16::GFP + rol-6(su1006)] ; fsEx400[Pgpa- } \\
\text { 4del6::odr-10g::SL2mCherry::unc-54-3'UTR+Punc-122::GFP] }\end{array}$ \\
\hline UR1291 & $\begin{array}{l}\text { tax-4(ok3771) III; him-5(e1490) V; ksIs2; fsEx550[Ptax-4::tax-4c::SL2::mCherry::unc-54 3' UTR + Pelt- } \\
\text { 2::GFP] }\end{array}$ \\
\hline UR1292 & $\begin{array}{l}\text { tax-4(ok3771) III; him-5(e1490) V; ks/s2 X; fsEx557[Pgpa-4::tax-4c::SL2::mCherry + Pssu-1::tax- } \\
\text { 4c:::SL2:::mCherry::unc-54 3' UTR + Pelt2::GFP line 1] }\end{array}$ \\
\hline UR1293 & $\begin{array}{l}\text { tax-4(ok3771) III; him-5(e1490) V; ks/s2 X; fsEx558[Pgpa-4::tax-4c::SL2::mCherry + Pssu-1::tax- } \\
\text { 4c:::SL2:::mCherry::unc-54 3' UTR + Pelt-2::GFP line 2] }\end{array}$ \\
\hline UR1294 & tax-2(p691) I; him-5(e1490) V; fsEx295 \\
\hline UR1295 & tax-2(p694) I; him-5(e1490) V; fsEx295 \\
\hline UR1296 & eat-2(ad465) II; him-5(e1490) V; fsEx295 \\
\hline UR1297 & ceh-36(ky640) X; pha-1(e2123) III; him-5(e1490) V; fsEx295 \\
\hline
\end{tabular}




\begin{tabular}{|l|l|}
\hline UR1298 & him-5(e1490) V; Is[Pstr-1::mCasp1]; fsEx295 \\
\hline UR1299 & him-8(e1489) IV; fsEx295 \\
\hline UR1300 & him-8(e1489) IV; qrls2[Psra-9::mCasp1] V; fsEx295 \\
\hline UR1301 & $\begin{array}{l}\text { tax-4(ok3771); him-5(e1490) V; kyls53 X; fsEx576[Pops-1-::tax-4c::SL2::mCherry::unc-54 3' UTR + Pelt- } \\
\text { 2::GFP line 1] }\end{array}$ \\
\hline UR1302 & $\begin{array}{l}\text { tax-4(ok3771); him-5(e1490) V; kyls53 X; fsEx577[Pops-1-::tax-4c::SL2::mCherry::unc-54 3' UTR + Pelt- } \\
\text { 2::GFP line 2] }\end{array}$ \\
\hline UR1303 & him-5(e1490) V; kyls53 X;fsls15[Prab-3::fem-3::SL2::mCherry::unc-54 3' UTR + Punc-122::GFP] \\
\hline UR1324 & tax-4(ok3771) III; him-5(e1490) V \\
\hline UR1325 & daf-16(mgDf47) I; tax-4(ok3771) III; him-5(e1490) V \\
\hline
\end{tabular}

552 TABLE S2. Primer List.

\begin{tabular}{|c|c|c|}
\hline $\begin{array}{l}\text { Primer } \\
\text { Name }\end{array}$ & Use & Sequence \\
\hline \multicolumn{3}{|c|}{ Gateway cloning } \\
\hline LW01 & $\operatorname{tax}-4 \mathrm{c} F$ & ggggacaagtttgtacaaaaaagcaggctatgtcaacggcggaacc \\
\hline LW02 & $\operatorname{tax}-4 c \mathrm{R}$ & ggggaccactttgtacaagaaagctgggtctatttgagcaaggattcagattc \\
\hline LW03 & daf-16f cDNA F & ggggacaagtttgtacaaaaaagcaggctatgcaagcgtggaactgtc \\
\hline LW04 & daf-16f cDNA R & ggggaccactttgtacaagaaagctgggtttacaaatcaaaatgaatatgctgc \\
\hline LW05 & Pdaf-8 F & ggggacaactttgtatagaaaagttgttgttagatgagcagtggtc \\
\hline LW06 & Pdaf-8 R & ggggactgcttttttgtacaaacttggttttaaaactgaaatttcgaagaa \\
\hline LW07 & Ptdc-1 F & ggggacaactttgtatagaaaagttgcggaaatttcaggtaaccaaagaac \\
\hline LW08 & Ptdc-1 R & ggggactgcttttttgtacaaacttgcgtaaaccatttgggcggt \\
\hline \multicolumn{3}{|c|}{ Construction of Podr-10 reporters } \\
\hline LW09 & fragment 1 podr-10 f1 & ggacgtgcgaaatagcattgg \\
\hline LW10 & $\begin{array}{l}\text { fragment } 1 \text { podr-10 } \mathrm{r} 1 \mathrm{w} / \\
\text { daf-16 site mutation }\end{array}$ & ttacatttaaatcaatattttggggacgaatatggcctaaaagttgcgtg \\
\hline LW11 & $\begin{array}{l}\text { fragment } 2 \text { podr- } 10 \mathrm{f} 2 \mathrm{w} / \\
\text { daf-16 stie mutation }\end{array}$ & ccatattcgtccccaaaatattgatttaaatgtaatttttcgaactttaattc \\
\hline LW12 & $\begin{array}{l}\text { fragment } 2 \text { podr-10 r2 w/ } \\
\text { gfp overhang }\end{array}$ & aagttcttctcctttactcatccgacatggagctgtaaggtatc \\
\hline LW13 & fragment $3 \mathrm{gfp} \mathrm{f} 3$ & atgagtaaaggagaagaacttttcactgg \\
\hline LW14 & fragment $3 \mathrm{gfp} \mathrm{r} 3$ & tatcaacttctactcaccttccactg \\
\hline
\end{tabular}

555 


\section{REFERENCES}

558

559

560

561

562

563

564

565

566

567

568

569

570

571

572

573

574

575

576

577

578

579

580

581

582

583

584

585

586

587

588

589

590

591

592
1. Root, C.M., Ko, K.I., Jafari, A., and Wang, J.W. (2011). Presynaptic facilitation by neuropeptide signaling mediates odor-driven food search. Cell 145, 133-144.

2. Inagaki, H.K., Ben-Tabou de-Leon, S., Wong, A.M., Jagadish, S., Ishimoto, H., Barnea, G., Kitamoto, T., Axel, R., and Anderson, D.J. (2012). Visualizing neuromodulation in vivo: TANGO-mapping of dopamine signaling reveals appetite control of sugar sensing. Cell 148, 583-595.

3. Ko, K.I., Root, C.M., Lindsay, S.A., Zaninovich, O.A., Shepherd, A.K., Wasserman, S.A., Kim, S.M., and Wang, J.W. (2015). Starvation promotes concerted modulation of appetitive olfactory behavior via parallel neuromodulatory circuits. Elife 4, e08298.

4. Devineni, A.V., Sun, B., Zhukovskaya, A., and Axel, R. (2019). Acetic acid activates distinct taste pathways in Drosophila to elicit opposing, state-dependent feeding responses. Elife 8, e47677.

5. Ryu, L., Cheon, Y., Huh, Y.H., Pyo, S., Chinta, S., Choi, H., Butcher, R.A., and Kim, K. (2018). Feeding state regulates pheromone-mediated avoidance behavior via the insulin signaling pathway in Caenorhabditis elegans. The EMBO journal 37, e98402.

6. Gruner, M., Grubbs, J., McDonagh, A., Valdes, D., Winbush, A., and van der Linden, A.M. (2016). Cell-Autonomous and Non-Cell-Autonomous Regulation of a Feeding State-Dependent Chemoreceptor Gene via MEF-2 and bHLH Transcription Factors. PLoS genetics 12, e1006237.

7. Gruner, M., Nelson, D., Winbush, A., Hintz, R., Ryu, L., Chung, S.H., Kim, K., Gabel, C.V., and van der Linden, A.M. (2014). Feeding state, insulin and NPR-1 modulate chemoreceptor gene expression via integration of sensory and circuit inputs. PLoS genetics 10, e1004707.

8. Ezcurra, M., Tanizawa, Y., Swoboda, P., and Schafer, W.R. (2011). Food sensitizes C. elegans avoidance behaviours through acute dopamine signalling. The EMBO journal 30, 1110-1122.

9. Ezcurra, M., Walker, D.S., Beets, I., Swoboda, P., and Schafer, W.R. (2016). Neuropeptidergic Signaling and Active Feeding State Inhibit Nociception in Caenorhabditis elegans. The Journal of neuroscience : the official journal of the Society for Neuroscience 36, 3157-3169.

10. Chao, M.Y., Komatsu, H., Fukuto, H.S., Dionne, H.M., and Hart, A.C. (2004). Feeding status and serotonin rapidly and reversibly modulate a Caenorhabditis elegans chemosensory circuit. Proceedings of the National Academy of Sciences of the United States of America 101, 1551215517.

11. Harris, G., Mills, H., Wragg, R., Hapiak, V., Castelletto, M., Korchnak, A., and Komuniecki, R.W. (2010). The monoaminergic modulation of sensory-mediated aversive responses in Caenorhabditis elegans requires glutamatergic/peptidergic cotransmission. The Journal of neuroscience : the official journal of the Society for Neuroscience 30, 7889-7899.

12. Ryan, D.A., Miller, R.M., Lee, K., Neal, S.J., Fagan, K.A., Sengupta, P., and Portman, D.S. (2014). Sex, age, and hunger regulate behavioral prioritization through dynamic modulation of chemoreceptor expression. Current biology : CB 24, 2509-2517. 
13. Tong, J., Mannea, E., Aime, P., Pfluger, P.T., Yi, C.X., Castaneda, T.R., Davis, H.W., Ren, X., Pixley, S., Benoit, S., et al. (2011). Ghrelin enhances olfactory sensitivity and exploratory sniffing in rodents and humans. The Journal of neuroscience : the official journal of the Society for Neuroscience 31, 5841-5846.

14. Tucker, K., Cho, S., Thiebaud, N., Henderson, M.X., and Fadool, D.A. (2013). Glucose sensitivity of mouse olfactory bulb neurons is conveyed by a voltage-gated potassium channel. J Physiol 591, 2541-2561.

15. Gaudry, Q., and Kristan, W.B., Jr. (2009). Behavioral choice by presynaptic inhibition of tactile sensory terminals. Nature neuroscience 12, 1450-1457.

16. Palouzier-Paulignan, B., Lacroix, M.C., Aime, P., Baly, C., Caillol, M., Congar, P., Julliard, A.K., Tucker, K., and Fadool, D.A. (2012). Olfaction under metabolic influences. Chem Senses 37, 769797.

17. Sengupta, P. (2013). The belly rules the nose: feeding state-dependent modulation of peripheral chemosensory responses. Curr Opin Neurobiol 23, 68-75.

18. Mowrey, W.R., and Portman, D.S. (2012). Sex differences in behavioral decision-making and the modulation of shared neural circuits. Biology of sex differences 3,8 .

19. White, J., Nicholas, T., Gritton, J., Truong, L., Davidson, E., and Jorgensen, E. (2007). The sensory circuitry for sexual attraction in C. elegans males. Current biology : CB 17, 1847-1857.

20. Fagan, K.A., and Portman, D.S. (2014). Sexual modulation of neural circuits and behavior in Caenorhabditis elegans. Seminars in cell \& developmental biology 33C, 3-9.

21. Lee, K., and Portman, D. (2007). Neural sex modifies the function of a C. elegans sensory circuit. Current biology : CB 17, 1858-1863.

22. Jang, H., Kim, K., Neal, S.J., Macosko, E., Kim, D., Butcher, R.A., Zeiger, D.M., Bargmann, C.I., and Sengupta, P. (2012). Neuromodulatory state and sex specify alternative behaviors through antagonistic synaptic pathways in C. elegans. Neuron 75, 585-592.

23. Lipton, J., Kleemann, G., Ghosh, R., Lints, R., and Emmons, S.W. (2004). Mate searching in Caenorhabditis elegans: a genetic model for sex drive in a simple invertebrate. The Journal of neuroscience : the official journal of the Society for Neuroscience 24, 7427-7434.

24. Zhou, S., Stone, E.A., Mackay, T.F., and Anholt, R.R. (2009). Plasticity of the chemoreceptor repertoire in Drosophila melanogaster. PLoS genetics 5, e1000681.

25. Bray, S., and Amrein, H. (2003). A putative Drosophila pheromone receptor expressed in malespecific taste neurons is required for efficient courtship. Neuron 39, 1019-1029.

26. Meunier, N., Ferveur, J.F., and Marion-Poll, F. (2000). Sex-specific non-pheromonal taste receptors in Drosophila. Current biology : CB 10, 1583-1586. 
27. Hussain, A., Ucpunar, H.K., Zhang, M., Loschek, L.F., and Grunwald Kadow, I.C. (2016). Neuropeptides Modulate Female Chemosensory Processing upon Mating in Drosophila. PLoS biology 14 , e1002455.

28. Xu, P.S., Lee, D., and Holy, T.E. (2016). Experience-Dependent Plasticity Drives Individual Differences in Pheromone-Sensing Neurons. Neuron 91, 878-892.

29. Dey, S., Chamero, P., Pru, J.K., Chien, M.S., Ibarra-Soria, X., Spencer, K.R., Logan, D.W., Matsunami, H., Peluso, J.J., and Stowers, L. (2015). Cyclic Regulation of Sensory Perception by a Female Hormone Alters Behavior. Cell 161, 1334-1344.

30. Kanageswaran, N., Nagel, M., Scholz, P., Mohrhardt, J., Gisselmann, G., and Hatt, H. (2016). Modulatory Effects of Sex Steroids Progesterone and Estradiol on Odorant Evoked Responses in Olfactory Receptor Neurons. PloS one 11, e0159640.

31. Kass, M.D., Czarnecki, L.A., Moberly, A.H., and McGann, J.P. (2017). Differences in peripheral sensory input to the olfactory bulb between male and female mice. Sci Rep 7, 45851.

32. Barrios, A. (2014). Exploratory decisions of the Caenorhabditis elegans male: a conflict of two drives. Seminars in cell \& developmental biology 33, 10-17.

33. Barrios, A., Nurrish, S., and Emmons, S.W. (2008). Sensory regulation of C. elegans male matesearching behavior. Current biology : CB 18, 1865-1871.

34. Barrios, A., Ghosh, R., Fang, C., Emmons, S.W., and Barr, M.M. (2012). PDF-1 neuropeptide signaling modulates a neural circuit for mate-searching behavior in C. elegans. Nature neuroscience $15,1675-1682$.

35. Hilbert, Z.A., and Kim, D.H. (2018). PDF-1 neuropeptide signaling regulates sexually dimorphic gene expression in shared sensory neurons of C. elegans. Elife 7, e36547.

36. Sengupta, P., Chou, J.H., and Bargmann, C.I. (1996). odr-10 encodes a seven transmembrane domain olfactory receptor required for responses to the odorant diacetyl. Cell 84, 899-909.

37. Lawson, H., Vuong, E., Miller, R.M., Kiontke, K., Fitch, D.H., and Portman, D.S. (2019). The Makorin lep-2 and the IncRNA lep-5 regulate lin-28 to schedule sexual maturation of the $\mathrm{C}$. elegans nervous system. Elife 8.

38. Hilbert, Z.A., and Kim, D.H. (2017). Sexually dimorphic control of gene expression in sensory neurons regulates decision-making behavior in C. elegans. Elife 6, e21166.

39. Hahm, J.H., Kim, S., DiLoreto, R., Shi, C., Lee, S.J., Murphy, C.T., and Nam, H.G. (2015). C. elegans maximum velocity correlates with healthspan and is maintained in worms with an insulin receptor mutation. Nat Commun 6, 8919.

40. Ren, P., Lim, C.S., Johnsen, R., Albert, P.S., Pilgrim, D., and Riddle, D.L. (1996). Control of C. elegans larval development by neuronal expression of a TGF-beta homolog. Science 274, 1389-1391. 
661

662

663

664

665

666

667

668

669

670

671

672

673

674

675

676

677

678

679

680

681

682

683

684

685

686

687

688

689

690

691

692

693

694

695

41. Schackwitz, W.S., Inoue, T., and Thomas, J.H. (1996). Chemosensory neurons function in parallel to mediate a pheromone response in C. elegans. Neuron 17, 719-728.

42. Greer, E.R., Perez, C.L., Van Gilst, M.R., Lee, B.H., and Ashrafi, K. (2008). Neural and molecular dissection of a $C$. elegans sensory circuit that regulates fat and feeding. Cell metabolism $8,118-$ 131.

43. You, Y.J., Kim, J., Raizen, D.M., and Avery, L. (2008). Insulin, CGMP, and TGF-beta signals regulate food intake and quiescence in C. elegans: a model for satiety. Cell metabolism 7, 249-257.

44. Meisel, J.D., Panda, O., Mahanti, P., Schroeder, F.C., and Kim, D.H. (2014). Chemosensation of bacterial secondary metabolites modulates neuroendocrine signaling and behavior of $\mathrm{C}$. elegans. Cell 159, 267-280.

45. Chang, A.J., Chronis, N., Karow, D.S., Marletta, M.A., and Bargmann, C.I. (2006). A distributed chemosensory circuit for oxygen preference in C. elegans. PLoS biology 4, e274.

46. Gallagher, T., Kim, J., Oldenbroek, M., Kerr, R., and You, Y.J. (2013). ASI Regulates Satiety Quiescence in C. elegans. The Journal of neuroscience : the official journal of the Society for Neuroscience 33, 9716-9724.

47. Milward, K., Busch, K.E., Murphy, R.J., de Bono, M., and Olofsson, B. (2011). Neuronal and molecular substrates for optimal foraging in Caenorhabditis elegans. Proceedings of the National Academy of Sciences of the United States of America 108, 20672-20677.

48. Dalfo, D., Michaelson, D., and Hubbard, E.J. (2012). Sensory regulation of the C. elegans germline through TGF-beta-dependent signaling in the niche. Current biology : CB 22, 712-719.

49. Entchev, E.V., Patel, D.S., Zhan, M., Steele, A.J., Lu, H., and Ch'ng, Q. (2015). A gene-expressionbased neural code for food abundance that modulates lifespan. Elife 4, e06259.

50. Singh, J., and Aballay, A. (2019). Microbial Colonization Activates an Immune Fight-and-Flight Response via Neuroendocrine Signaling. Dev Cell 49, 89-99 e84.

51. Neal, S.J., Takeishi, A., O'Donnell, M.P., Park, J., Hong, M., Butcher, R.A., Kim, K., and Sengupta, P. (2015). Feeding state-dependent regulation of developmental plasticity via CaMKI and neuroendocrine signaling. Elife 4, e10110.

52. McKnight, K., Hoang, H.D., Prasain, J.K., Brown, N., Vibbert, J., Hollister, K.A., Moore, R., Ragains, J.R., Reese, J., and Miller, M.A. (2014). Neurosensory perception of environmental cues modulates sperm motility critical for fertilization. Science $344,754-757$.

53. Shaw, W.M., Luo, S., Landis, J., Ashraf, J., and Murphy, C.T. (2007). The C. elegans TGF-beta Dauer pathway regulates longevity via insulin signaling. Current biology : CB 17, 1635-1645.

54. Nolan, K.M., Sarafi-Reinach, T.R., Horne, J.G., Saffer, A.M., and Sengupta, P. (2002). The DAF-7 TGF-beta signaling pathway regulates chemosensory receptor gene expression in C. elegans. Genes Dev 16, 3061-3073. 
55. Liu, T., Zimmerman, K.K., and Patterson, G.I. (2004). Regulation of signaling genes by TGFbeta during entry into dauer diapause in C. elegans. BMC Dev Biol 4, 11.

56. Narasimhan, S.D., Yen, K., Bansal, A., Kwon, E.S., Padmanabhan, S., and Tissenbaum, H.A. (2011). PDP-1 links the TGF-beta and IIS pathways to regulate longevity, development, and metabolism. PLoS genetics 7, e1001377.

57. Lee, R.Y., Hench, J., and Ruvkun, G. (2001). Regulation of C. elegans DAF-16 and its human ortholog FKHRL1 by the daf-2 insulin-like signaling pathway. Current biology : CB 11, 1950-1957.

58. Gumienny, T.L., and Savage-Dunn, C. (2013). TGF-beta signaling in C. elegans. WormBook : the online review of $\mathrm{C}$. elegans biology, 1-34.

59. Gruninger, T., Gualberto, D., LeBoeuf, B., and Garcia, L. (2006). Integration of male mating and feeding behaviors in Caenorhabditis elegans. J Neurosci S 26, 169-179.

60. Raizen, D.M., Lee, R.Y., and Avery, L. (1995). Interacting genes required for pharyngeal excitation by motor neuron MC in Caenorhabditis elegans. Genetics 141, 1365-1382.

61. Coburn, C.M., and Bargmann, C.I. (1996). A putative cyclic nucleotide-gated channel is required for sensory development and function in C. elegans. Neuron 17, 695-706.

62. Komatsu, H., Mori, I., Rhee, J., Akaike, N., and Ohshima, Y. (1996). Mutations in a cyclic nucleotidegated channel lead to abnormal thermosensation and chemosensation in C. elegans. Neuron 17, 707-718.

63. Coburn, C.M., Mori, I., Ohshima, Y., and Bargmann, C.I. (1998). A cyclic nucleotide-gated channel inhibits sensory axon outgrowth in larval and adult Caenorhabditis elegans: a distinct pathway for maintenance of sensory axon structure. Development 125, 249-258.

64. Bretscher, A.J., Kodama-Namba, E., Busch, K.E., Murphy, R.J., Soltesz, Z., Laurent, P., and de Bono, $M$. (2011). Temperature, oxygen, and salt-sensing neurons in $C$. elegans are carbon dioxide sensors that control avoidance behavior. Neuron 69, 1099-1113.

65. Zaslaver, A., Liani, I., Shtangel, O., Ginzburg, S., Yee, L., and Sternberg, P.W. (2015). Hierarchical sparse coding in the sensory system of Caenorhabditis elegans. Proceedings of the National Academy of Sciences of the United States of America 112, 1185-1189.

66. Toates, F.M. (1986). Motivational systems, (Cambridge Cambridgeshire ; New York: Cambridge University Press).

67. Worthy, S.E., Haynes, L., Chambers, M., Bethune, D., Kan, E., Chung, K., Ota, R., Taylor, C.J., and Glater, E.E. (2018). Identification of attractive odorants released by preferred bacterial food found in the natural habitats of C. elegans. PloS one 13, e0201158.

68. Daniels, S.A., Ailion, M., Thomas, J.H., and Sengupta, P. (2000). egl-4 acts through a transforming growth factor-beta/SMAD pathway in Caenorhabditis elegans to regulate multiple neuronal circuits in response to sensory cues. Genetics 156, 123-141. 
69. Murphy, C.T., and Hu, P.J. (2013). Insulin/insulin-like growth factor signaling in C. elegans. WormBook : the online review of $\mathrm{C}$. elegans biology, 1-43.

70. Vowels, J.J., and Thomas, J.H. (1992). Genetic analysis of chemosensory control of dauer formation in Caenorhabditis elegans. Genetics 130, 105-123.

71. Ogg, S., Paradis, S., Gottlieb, S., Patterson, G.I., Lee, L., Tissenbaum, H.A., and Ruvkun, G. (1997). The Fork head transcription factor DAF-16 transduces insulin-like metabolic and longevity signals in C. elegans. Nature 389, 994-999.

72. Hu, P.J. (2007). Dauer. WormBook : the online review of C. elegans biology, 1-19.

73. Tomioka, M., Adachi, T., Suzuki, H., Kunitomo, H., Schafer, W.R., and lino, Y. (2006). The insulin/PI 3-kinase pathway regulates salt chemotaxis learning in Caenorhabditis elegans. Neuron 51, 613625.

74. Wan, X., Zhou, Y., Chan, C.M., Yang, H., Yeung, C., and Chow, K.L. (2019). SRD-1 in AWA neurons is the receptor for female volatile sex pheromones in C. elegans males. EMBO Rep 20, e46288.

75. Peckol, E.L., Troemel, E.R., and Bargmann, C.I. (2001). Sensory experience and sensory activity regulate chemosensory receptor gene expression in Caenorhabditis elegans. Proceedings of the National Academy of Sciences of the United States of America 98, 11032-11038.

76. Chen, D., Sitaraman, D., Chen, N., Jin, X., Han, C., Chen, J., Sun, M., Baker, B.S., Nitabach, M.N., and Pan, Y. (2017). Genetic and neuronal mechanisms governing the sex-specific interaction between sleep and sexual behaviors in Drosophila. Nat Commun 8, 154.

77. Beckwith, E.J., Geissmann, Q., French, A.S., and Gilestro, G.F. (2017). Regulation of sleep homeostasis by sexual arousal. Elife 6, e27455.

78. Mowrey, W.R., Bennett, J.R., and Portman, D.S. (2014). Distributed Effects of Biological Sex Define Sex-Typical Motor Behavior in Caenorhabditis elegans. The Journal of neuroscience : the official journal of the Society for Neuroscience 34, 1579-1591.

79. Barr, M.M., Garcia, L.R., and Portman, D.S. (2018). Sexual Dimorphism and Sex Differences in Caenorhabditis elegans Neuronal Development and Behavior. Genetics 208, 909-935.

80. O'Donnell, M.P., Chao, P.H., Kammenga, J.E., and Sengupta, P. (2018). Rictor/TORC2 mediates gutto-brain signaling in the regulation of phenotypic plasticity in C. elegans. PLoS genetics 14, e1007213.

81. Cunningham, K.A., Hua, Z., Srinivasan, S., Liu, J., Lee, B.H., Edwards, R.H., and Ashrafi, K. (2012). AMP-activated kinase links serotonergic signaling to glutamate release for regulation of feeding behavior in C. elegans. Cell metabolism 16, 113-121.

82. Ahmadi, M., and Roy, R. (2016). AMPK acts as a molecular trigger to coordinate glutamatergic signals and adaptive behaviours during acute starvation. Elife 5, e16349. 
83. Dowen, R.H., Breen, P.C., Tullius, T., Conery, A.L., and Ruvkun, G. (2016). A microRNA program in the $C$. elegans hypodermis couples to intestinal mTORC2/PQM-1 signaling to modulate fat transport. Genes Dev 30, 1515-1528.

84. Soukas, A.A., Kane, E.A., Carr, C.E., Melo, J.A., and Ruvkun, G. (2009). Rictor/TORC2 regulates fat metabolism, feeding, growth, and life span in Caenorhabditis elegans. Genes Dev 23, 496-511.

85. Rhoades, J.L., Nelson, J.C., Nwabudike, I., Yu, S.K., McLachlan, I.G., Madan, G.K., Abebe, E., Powers, J.R., Colon-Ramos, D.A., and Flavell, S.W. (2019). ASICs Mediate Food Responses in an Enteric Serotonergic Neuron that Controls Foraging Behaviors. Cell 176, 85-97 e14.

86. Sze, J.Y., Victor, M., Loer, C., Shi, Y., and Ruvkun, G. (2000). Food and metabolic signalling defects in a Caenorhabditis elegans serotonin-synthesis mutant. Nature 403, 560-564.

87. Kang, C., and Avery, L. (2009). Systemic regulation of starvation response in Caenorhabditis elegans. Genes Dev 23, 12-17.

88. Srinivasan, S., Sadegh, L., Elle, I.C., Christensen, A.G., Faergeman, N.J., and Ashrafi, K. (2008). Serotonin regulates $C$. elegans fat and feeding through independent molecular mechanisms. Cell metabolism 7, 533-544.

89. Hussey, R., Stieglitz, J., Mesgarzadeh, J., Locke, T.T., Zhang, Y.K., Schroeder, F.C., and Srinivasan, S. (2017). Pheromone-sensing neurons regulate peripheral lipid metabolism in Caenorhabditis elegans. PLoS genetics 13, e1006806.

90. Palamiuc, L., Noble, T., Witham, E., Ratanpal, H., Vaughan, M., and Srinivasan, S. (2017). A tachykinin-like neuroendocrine signalling axis couples central serotonin action and nutrient sensing with peripheral lipid metabolism. Nat Commun 8, 14237.

91. Rengarajan, S., Yankura, K.A., Guillermin, M.L., Fung, W., and Hallem, E.A. (2019). Feeding state sculpts a circuit for sensory valence in Caenorhabditis elegans. Proceedings of the National Academy of Sciences of the United States of America 116, 1776-1781.

92. Oranth, A., Schultheis, C., Tolstenkov, O., Erbguth, K., Nagpal, J., Hain, D., Brauner, M., Wabnig, S., Steuer Costa, W., McWhirter, R.D., et al. (2018). Food Sensation Modulates Locomotion by Dopamine and Neuropeptide Signaling in a Distributed Neuronal Network. Neuron 100, 1414-1428 e1410.

93. Lopez-Cruz, A., Sordillo, A., Pokala, N., Liu, Q., McGrath, P.T., and Bargmann, C.I. (2019). Parallel Multimodal Circuits Control an Innate Foraging Behavior. Neuron 102, 407-419 e408.

94. Jordan, J.M., Hibshman, J.D., Webster, A.K., Kaplan, R.E.W., Leinroth, A., Guzman, R., Maxwell, C.S., Chitrakar, R., Bowman, E.A., Fry, A.L., et al. (2019). Insulin/IGF Signaling and Vitellogenin Provisioning Mediate Intergenerational Adaptation to Nutrient Stress. Current biology : CB 29, 2380-2388 e2385.

95. Hills, T., Brockie, P., and Maricq, A. (2004). Dopamine and glutamate control area-restricted search behavior in Caenorhabditis elegans. The Journal of neuroscience : the official journal of the Society for Neuroscience 24, 1217-1225. 
802

803

804

805

806

807

808

809

810

811

812

813

814

815

816

817

818

819

820

821

822

823

824

825

826

827

828

829

830

831

832

833

834

835
96. Cohen, M., Reale, V., Olofsson, B., Knights, A., Evans, P., and de Bono, M. (2009). Coordinated regulation of foraging and metabolism in C. elegans by RFamide neuropeptide signaling. Cell metabolism 9, 375-385.

97. Gruninger, T.R., Gualberto, D.G., and Garcia, L.R. (2008). Sensory perception of food and insulinlike signals influence seizure susceptibility. PLoS genetics 4, e1000117.

98. Correa, P.A., Gruninger, T., and García, L.R. (2015). DOP-2 D2-Like receptor regulates UNC-7 innexins to attenuate recurrent sensory motor neurons during $C$. elegans copulation. The Journal of neuroscience : the official journal of the Society for Neuroscience 35, 9990-10004.

99. Ben Arous, J., Laffont, S., and Chatenay, D. (2009). Molecular and sensory basis of a food related two-state behavior in C. elegans. PloS one 4, e7584.

100. Juozaityte, V., Pladevall-Morera, D., Podolska, A., Norgaard, S., Neumann, B., and Pocock, R. (2017). The ETS-5 transcription factor regulates activity states in Caenorhabditis elegans by controlling satiety. Proceedings of the National Academy of Sciences of the United States of America 114, E1651-E1658.

101. Fenk, L.A., and de Bono, M. (2017). Memory of recent oxygen experience switches pheromone valence in Caenorhabditis elegans. Proceedings of the National Academy of Sciences of the United States of America 114, 4195-4200.

102. Alcedo, J., and Kenyon, C. (2004). Regulation of C. elegans longevity by specific gustatory and olfactory neurons. Neuron 41, 45-55.

103. Stiernagle, T. (2006). Maintenance of C. elegans. WormBook : the online review of C. elegans biology, 1-11.

104. Schindelin, J., Arganda-Carreras, I., Frise, E., Kaynig, V., Longair, M., Pietzsch, T., Preibisch, S., Rueden, C., Saalfeld, S., Schmid, B., et al. (2012). Fiji: an open-source platform for biological-image analysis. Nat Methods 9, 676-682.

105. Pfaffl, M.W. (2001). A new mathematical model for relative quantification in real-time RT-PCR. Nucleic Acids Res 29, e45.

106. Hoogewijs, D., Houthoofd, K., Matthijssens, F., Vandesompele, J., and Vanfleteren, J.R. (2008). Selection and validation of a set of reliable reference genes for quantitative sod gene expression analysis in C. elegans. BMC Mol Biol 9, 9.

107. Bargmann, C.I., Hartwieg, E., and Horvitz, H.R. (1993). Odorant-selective genes and neurons mediate olfaction in C. elegans. Cell 74, 515-527.

108. Gibson, D.G., Young, L., Chuang, R.Y., Venter, J.C., Hutchison, C.A., and Smith, H.O. (2009). Enzymatic assembly of DNA molecules up to several hundred kilobases. Nat Methods 6, 343-345. 
A

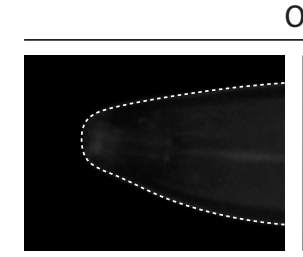

well-fed male
ODR-10::GFP (fsEx295)

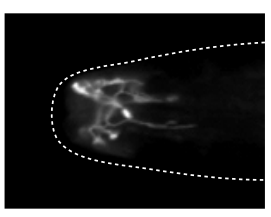

starved male

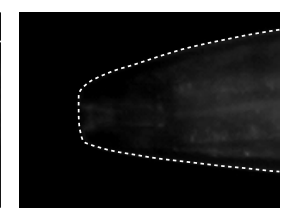

re-fed male
B ODR-10::GFP (fsEX295)

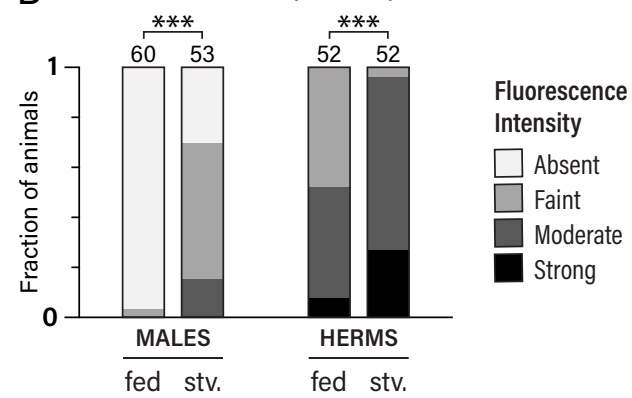

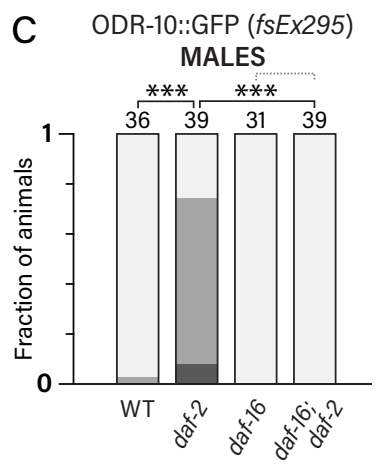

D Diacetyl chemotaxis MALES

E

ODR-10::GFP (fsEx295)
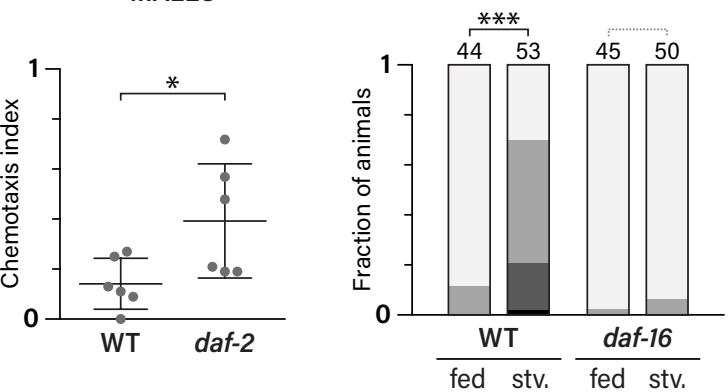

F

Diacetyl chemotaxis MALES

G ODR-10::GFP (fsEx295)

H ODR-10::GFP (fsEx295)

I
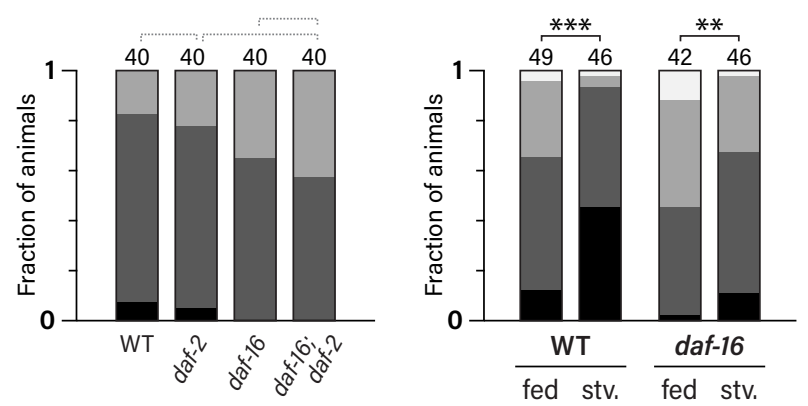

food availability

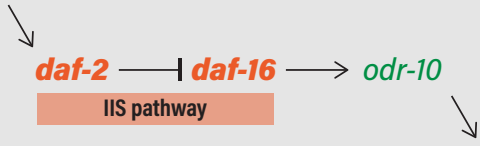

diacetyl chemotaxis food attraction

Figure 1. Insulin-like signaling regulates odr-10 expression males in response to feeding state. (A) Representative images of ODR-10::GFP fluorescence in a well fed male, a food-deprived ("starved") male, and a male re-fed after food deprivation. (B) ODR-10::GFP expression in WT fed and starved (stv) males and hermaphrodites, scored qualitatively on a four-point scale (see Methods). (C) ODR-10::GFP expression in male insulin-signaling mutants. (D) Chemotaxis to diacetyl (1:1000) in WT and daf-2 males. (E) ODR-10::GFP expression in fed and starved WT and daf16 males. (F) Chemotaxis to diacetyl (1:1000) in WT and daf-16 males either well-fed or starved for 12 hours. (G) ODR-10::GFP expression in hermaphrodite insulin-signaling mutants. (H) ODR-10::GFP expression in fed and starved WT and daf-16 hermaphrodites. (I) Pathway for the regulation of odr-10 by food availability and IIS. *0.01 < $p<0.05 ; * * 0.001<p<0.01 ; * * *<0.001$. Dotted gray brackets indicate $p>0.05$. 
A

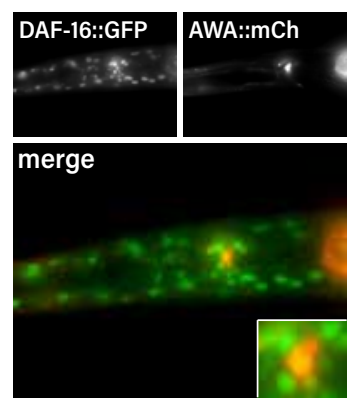

B

ODR-10::GFP $(f s E \times 295)$

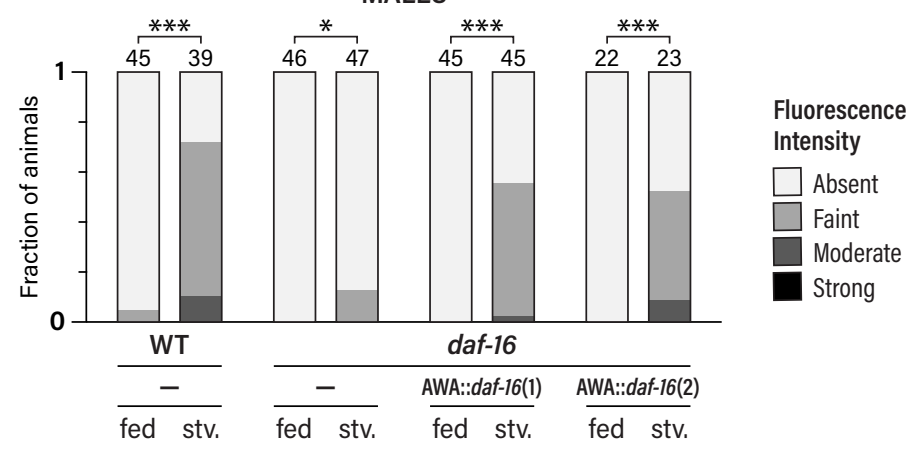

C

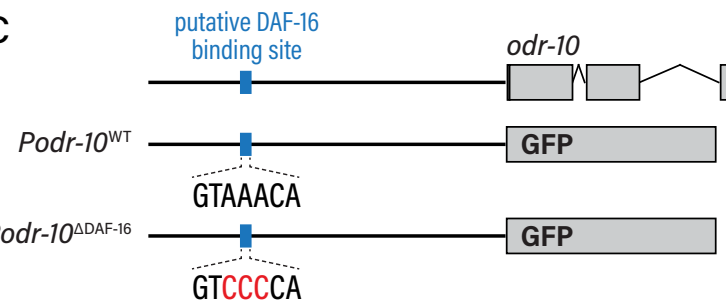

D

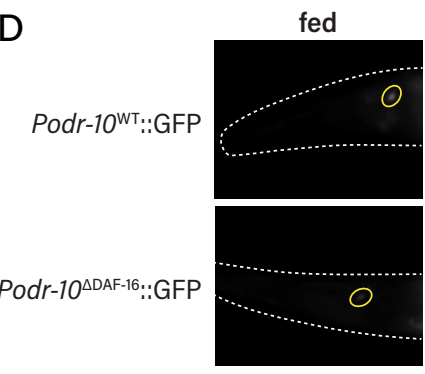

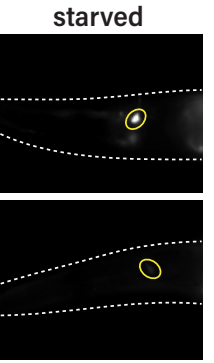

E Podr-10::GFP fluorescence intensity

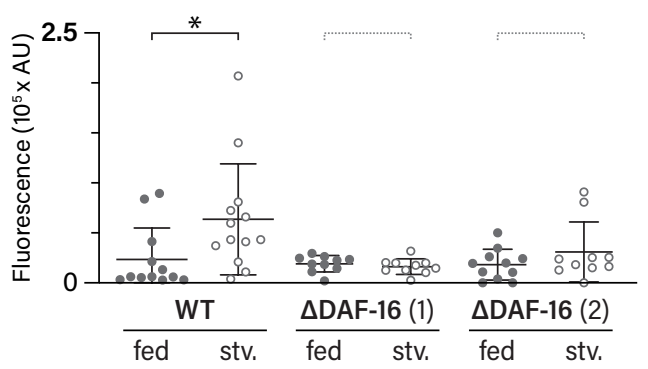

Figure 2. DAF-16 acts cell-autonomously, and likely directly, to regulate odr-10. (A) DAF-16::GFP and AWA::mCherry expression. Upper panels show individual channels; lower panel shows a merged, pseudocolored image with a higher-magnification inset. To bring about nuclear localization of DAF-16, this strain carried a daf- 2 mutation and was grown at $25^{\circ} \mathrm{C}$. (B) ODR-10::GFP expression in WT and daf16 fed and starved (stv) males, with or without (-) AWA::daf-16f transgenes. Results are shown for two independent lines. (C) Diagram depicting the odr-10 genomic locus (upper) and odr-10 transcriptional reporters (middle and lower) with wild-type and mutant versions of the putative DAF-16 binding site. (D) Representative images of Podr-10 $10^{W T}:$ GFP and Podr-10 ${ }^{\triangle D A F-16}::$ GFP in fed and starved males. Yellow ovals indicate the location of AWA. (E) Quantification of GFP fluorescence in Podr-10 ${ }^{W T}:: G F P$ and Podr-10 $10^{\triangle D A F-}$ ${ }^{16}:: G F P$ in fed and starved males. For the mutant reporter, results using two independent lines are shown. ${ }^{*} 0.01<p<0.05 ;{ }^{* *} 0.001<p<0.01 ;{ }^{* *} p<0.001$. Dotted gray brackets indicate $p>0.05$. 

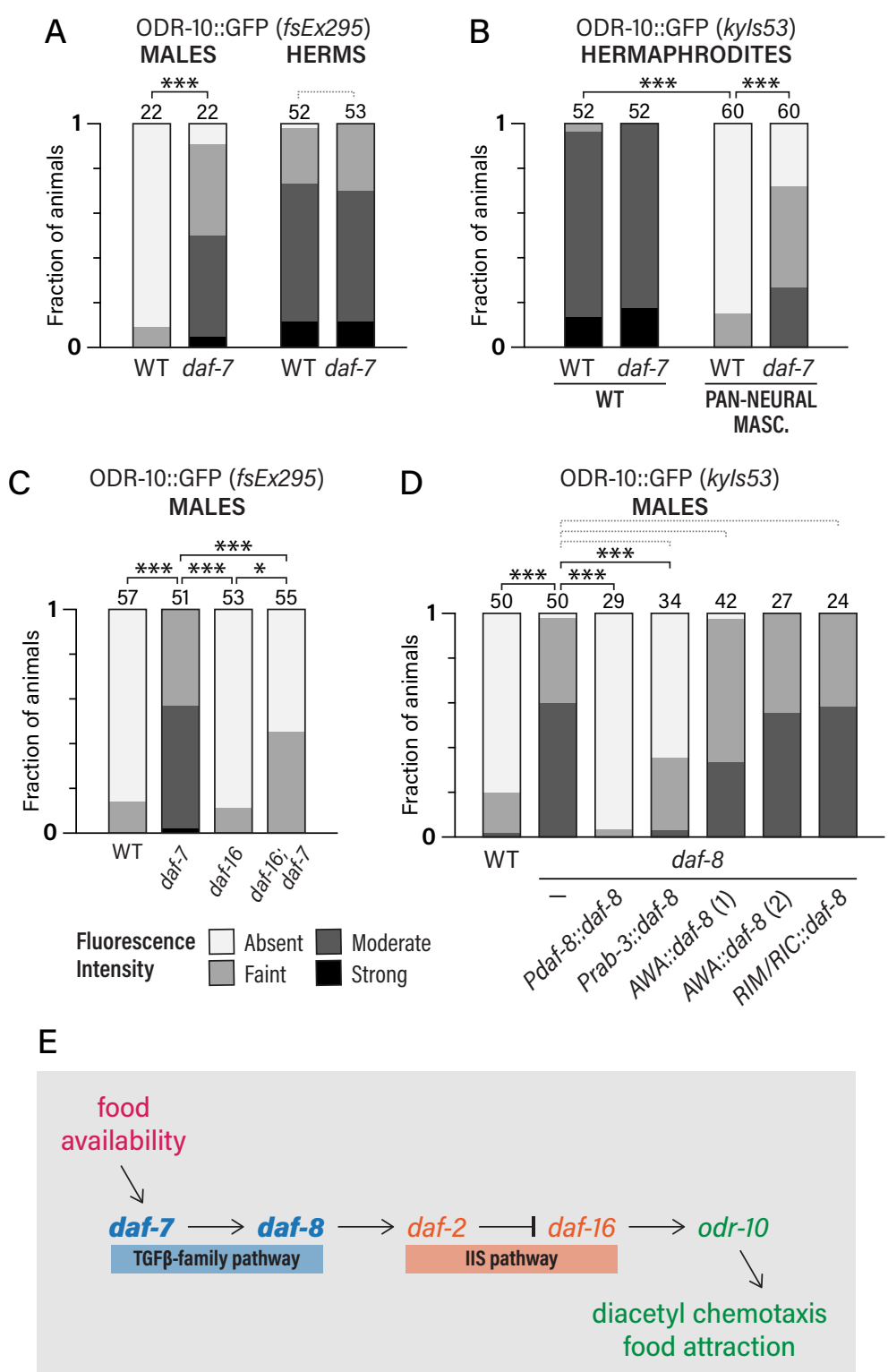

Figure 3. odr-10 is sex-specifically regulated by daf-7 TGF $\beta$. (A) ODR-10::GFP expression in WT and daf7 males and hermaphrodites. (B) ODR-10::GFP expression in WT and daf-7 hermaphrodites, with and without pan-neural masculinization by Prab-3::fem-3(+). (C) ODR-10::GFP expression in WT, daf-7, daf16, and daf-16; daf-7 mutant males. (D) ODR-10::GFP expression in WT and daf-8 mutants carrying the indicated daf-8 expression constructs. (E) Pathway for the regulation of $o d r-10$ by daf-7 and daf-2 (IIS) signaling. ${ }^{*} 0.01<p<0.05 ;{ }^{* *} 0.001<p<0.01 ;{ }^{* *} p<0.001$. Dotted gray brackets indicate $p>0.05$. 

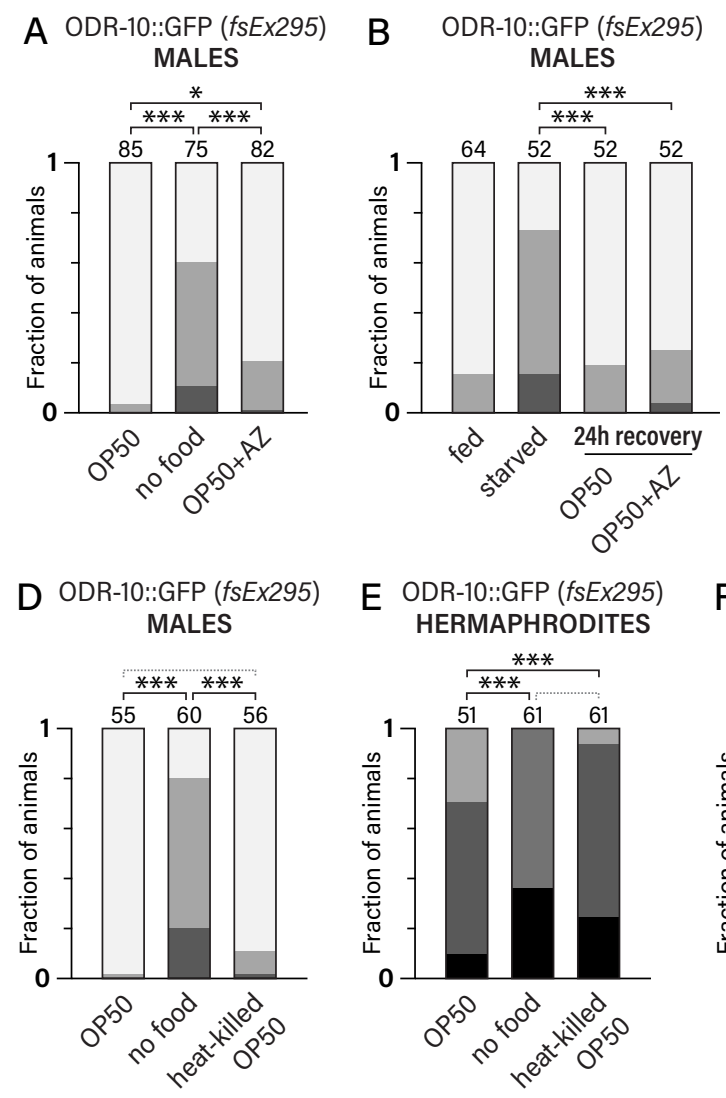

C ODR-10::GFP (fsEx295) HERMAPHRODITES

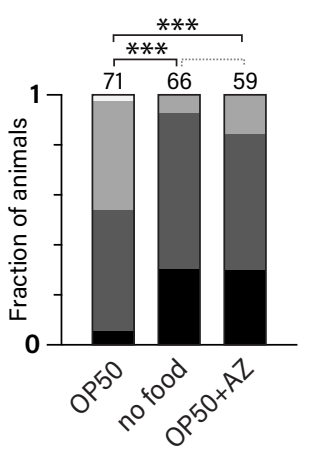

F

ODR-10::GFP (fsEx295)
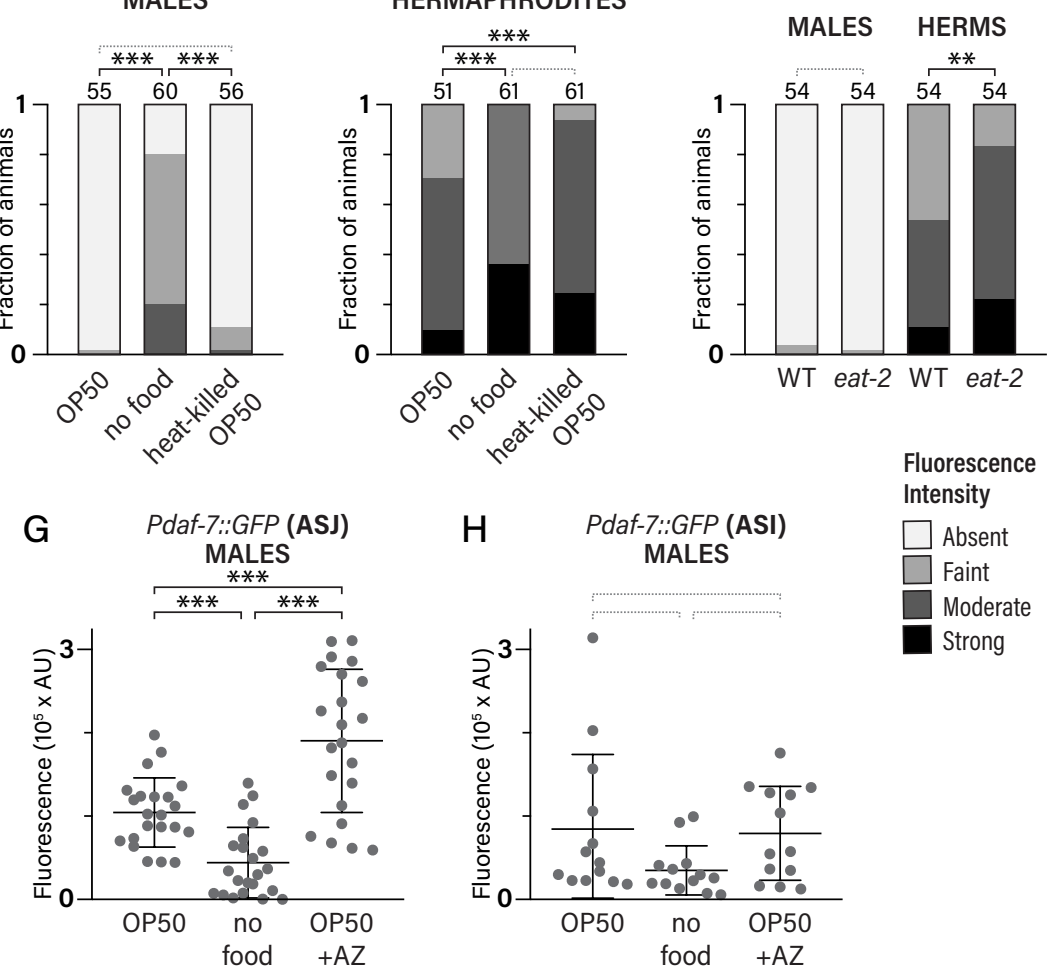

I

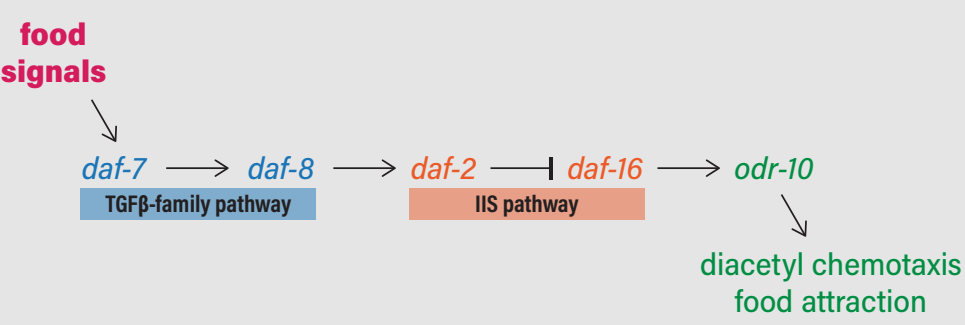

Figure 4. Sensory perception, not a metabolic cue, regulates odr-10 and daf-7 in males. (A) ODR-10::GFP expression in males cultured on E. coli OP50, without food, or on aztreonam-treated OP50 for 16-18 h. (B) ODR10::GFP expression in well-fed males, food-deprived males, and food-deprived males after $24 \mathrm{~h}$ recovery on control or aztreonam-treated OP50. (C) ODR-10::GFP expression in hermaphrodites cultured on OP50, without food, or on aztreonam-treated OP50 for 1618 h. (D-E) ODR-10::GFP expression in males (D) and hermaphrodites $(E)$ cultured on OP50, without food, or on heatkilled OP50 for 16-18 h. (F) ODR10::GFP expression in WT and eat-2 males and hermaphrodites. (G-H) Pdaf-7::GFP fluorescence intensity in ASJ $(G)$ and $A S I(H)$ on males cultured on OP50, without food, or on aztreonam-treated OP50. (I) Pathway for the regulation of $o d r-10$ by TGF $\beta$ and IIS in response to external food signals in males. ${ }^{*} 0.01<p<0.05$; $* * 0.001<p<0.01 ; * * * p<0.001$. Dotted gray brackets indicate $p>$ 0.05 . 

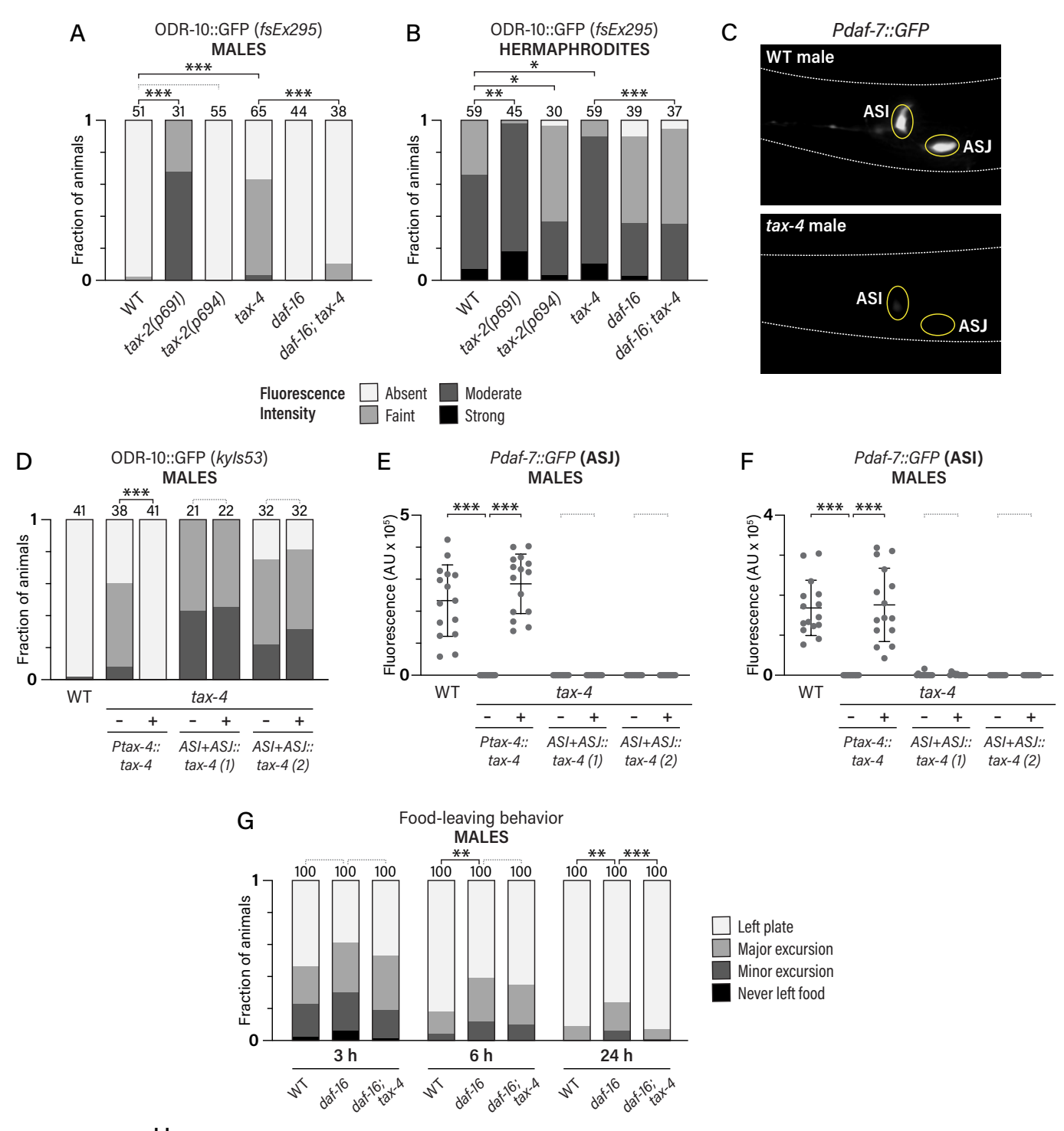

$\mathrm{H}$

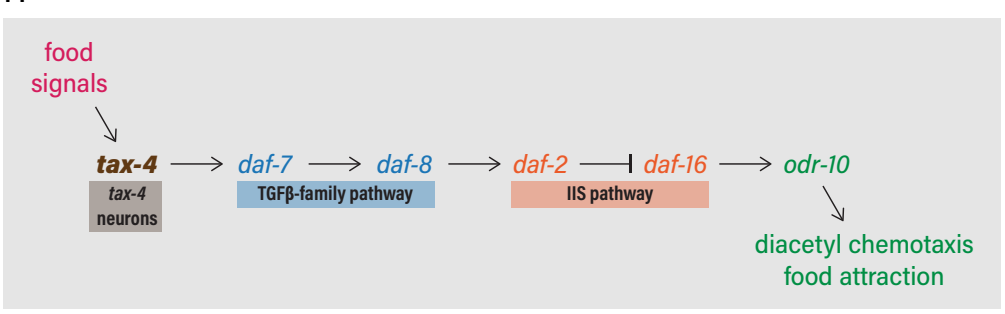

Figure 5. Signals from TAX-2/TAX-4 neurons regulate odr-10 expression in males. (A-B) ODR-10::GFP expression in tax-2, tax-4, daf-16, and daf-16; tax-4 males (A) and hermaphrodites (B). (C) Representative images of Pdaf7::GFP expression in WT and tax-4 males. Yellow ovals indicate the locations of ASI and ASJ. (D) ODR-10::GFP ( $k y / s 53)$ expression in WT and tax-4 males with the indicated tax-4 expression constructs. Two separate lines are shown for ASI+ASJ rescue. (E-F) Pdaf-7::GFP fluorescence in ASJ (E) and ASI (F) in tax-4 males with the indicated tax-4 expression constructs. Two separate lines are shown for ASI+ASJ rescue. (G) Food-leaving behavior of wildtype, tax-4, and daf-16; tax-4 mutant males at the indicated timepoints. For each plate, track patterns were scored manually using the four-point scale shown in the legend. (H) Pathway for the regulation of odr-10 by food signals, TGF $\beta$ signaling, and IIS in males. ${ }^{*} 0.01<p<0.05 ;{ }^{* *} 0.001<p<0.01 ;{ }^{* *} p<0.001$. Dotted gray brackets indicate $p$ $>0.05$. 

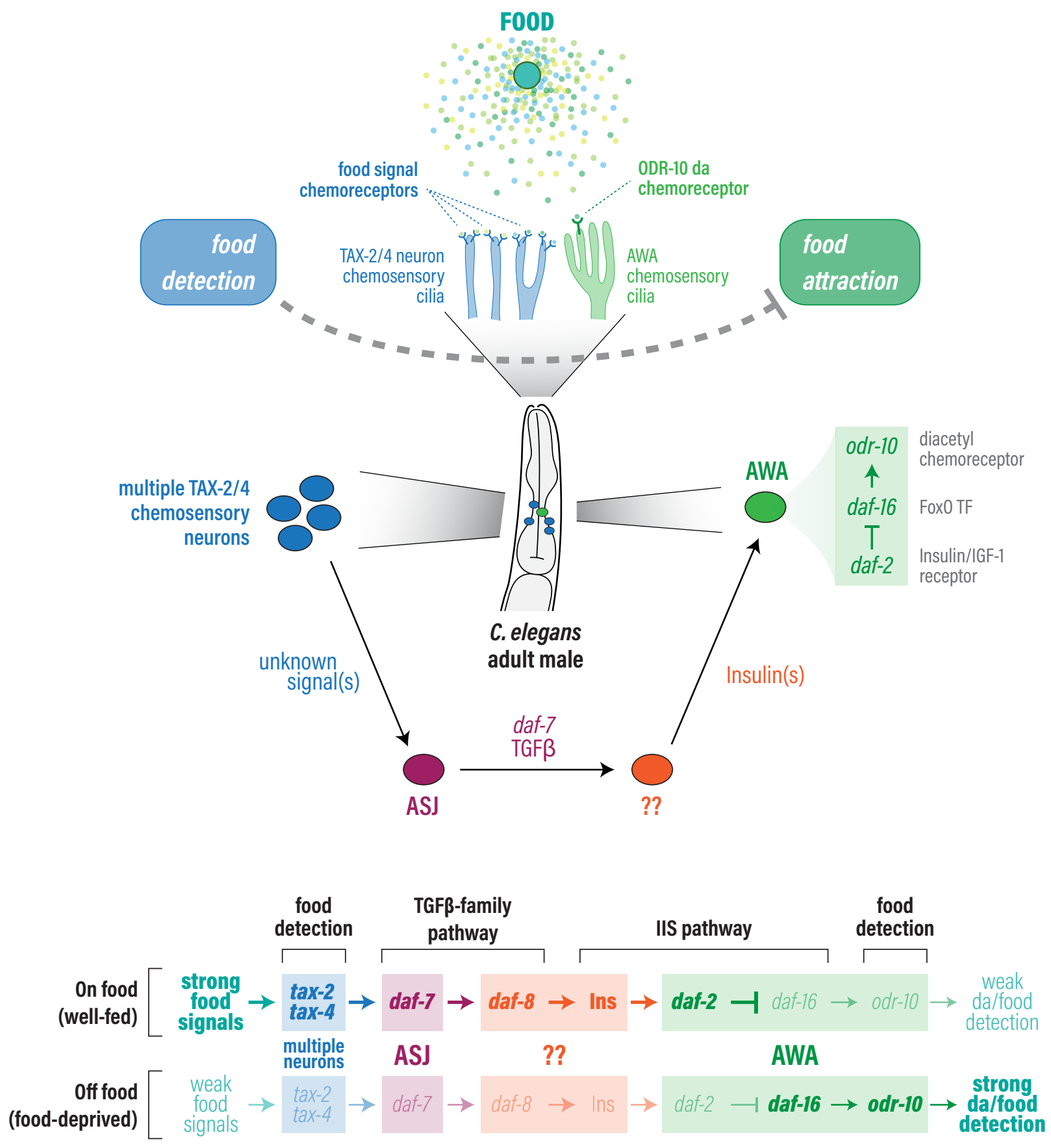

Figure 6. A sex-specific chemosensory feedback loop couples food detection, TGF $\beta$ signaling, and IIS to expression of the chemoreceptor odr-10 in adult $C$. elegans males. The model shows the proposed mechanism by which chemosensory information about food availability is transmitted through a neuroendocrine loop to regulate odr-10 in AWA. Pathways at the bottom depict the genetic architecture of the mechanism. See Discussion for details. 

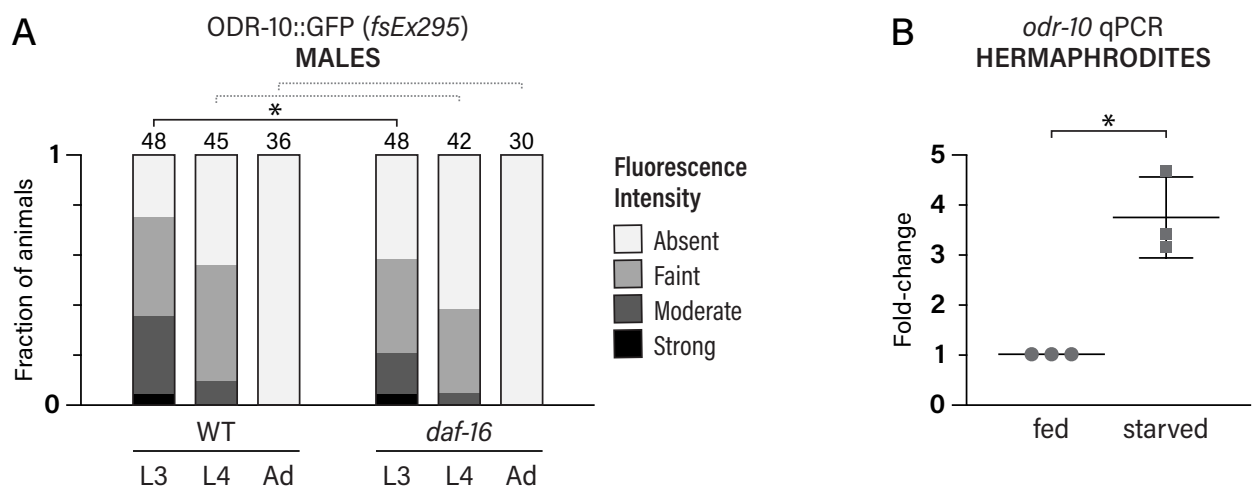

Figure S1. Related to Fig. 1. (A) ODR-10::GFP expression in L3, L4, and young adult WT and daf-16 males. (B) odr-10 mRNA levels as measured by qRT-PCR in fed and starved hermaphrodites, normalized to fed levels. ${ }^{*} 0.01<p<0.05 ;{ }^{* *} 0.001<p<0.01 ;{ }^{* *} p<0.001$. Dotted gray brackets indicate $p>0.05$.
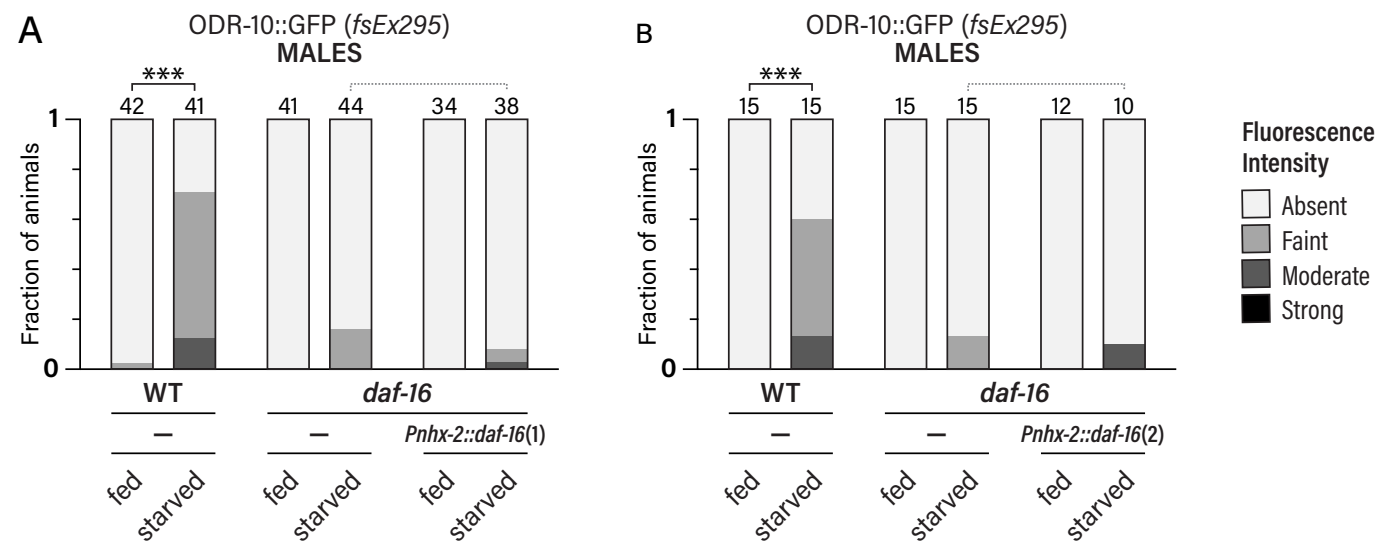

Figure S2. Related to Fig. 2. (A-B) ODR-10::GFP expression in WT and daf-16 fed and starved males with or without transgenes expressing daf-16f CDNA in the intestine. Independent experiments for two independent transgenic lines are shown: (A) line 1 ; (B) line $2 .{ }^{*} 0.01<p<0.05 ;{ }^{* *} 0.001<p<0.01 ;{ }^{* * *} p$ $<0.001$. Dotted gray brackets indicate $p>0.05$. 

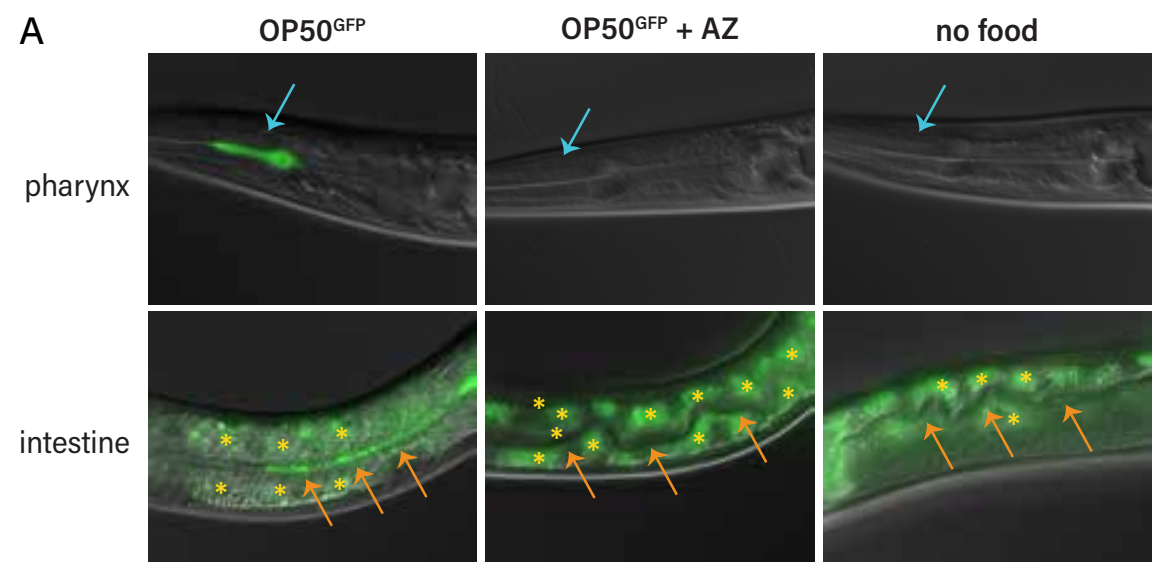

B

$$
\text { OP50 GFP INGESTION }
$$$$
\text { MALE }
$$

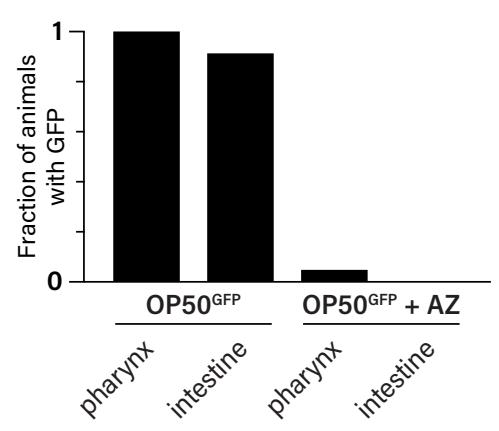

OP50GFP INGESTION
HERMAPHRODITE

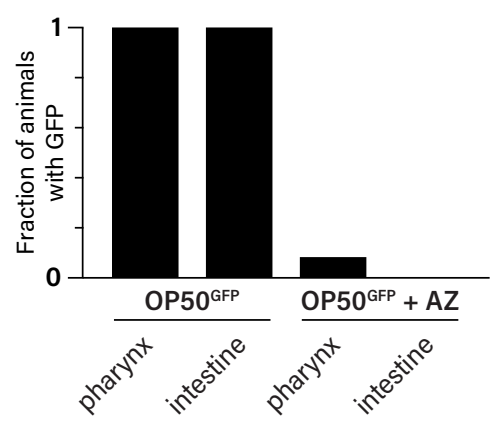

Figure S3. Related to Fig. 3. (A) Representative images of animals cultured with GFP-labeled E. coli OP50 $\left(\mathrm{OP} 50^{\mathrm{GFP}}\right)$, with aztreonam-treated OP50 ${ }^{\mathrm{GFP}}$, or without food for 16-18 h. Blue arrows show the pharyngeal lumen; orange arrows indicate intestinal lumen. Asterisks indicate autofluorescent granules in the intestine. (B-C) Percent of animals with GFP detectable in the pharynx and intestine of animals fed OP50 ${ }^{\text {GFP }}$ or aztreonam-treated OP50 ${ }^{\text {GFP }}$ for $16-18 \mathrm{~h}$. 


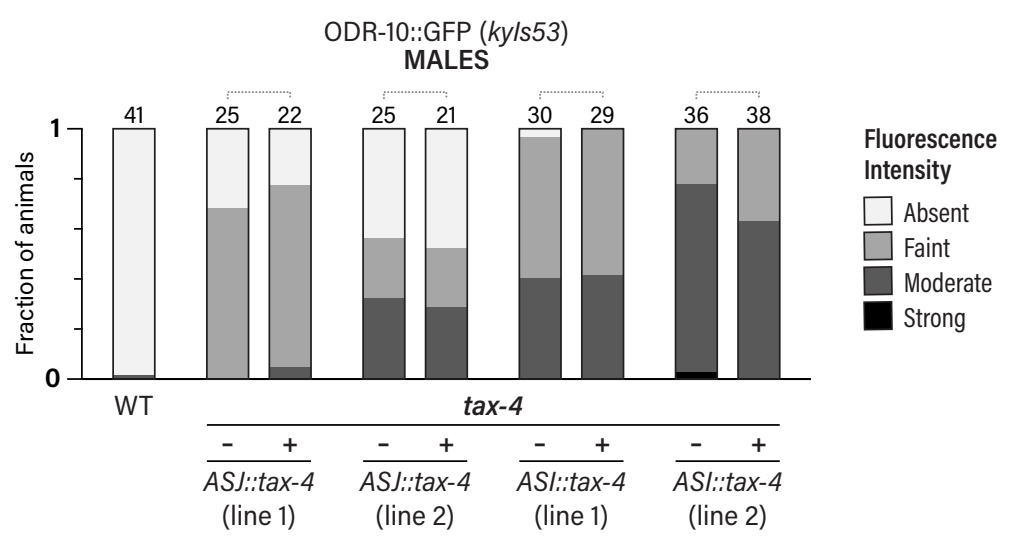

Figure S4. Related to Fig. 4. ODR-10::GFP (kyls53) expression in WT and tax-4 males with the indicated tax-4 expression constructs. Dotted gray brackets indicate $p>0.05$.
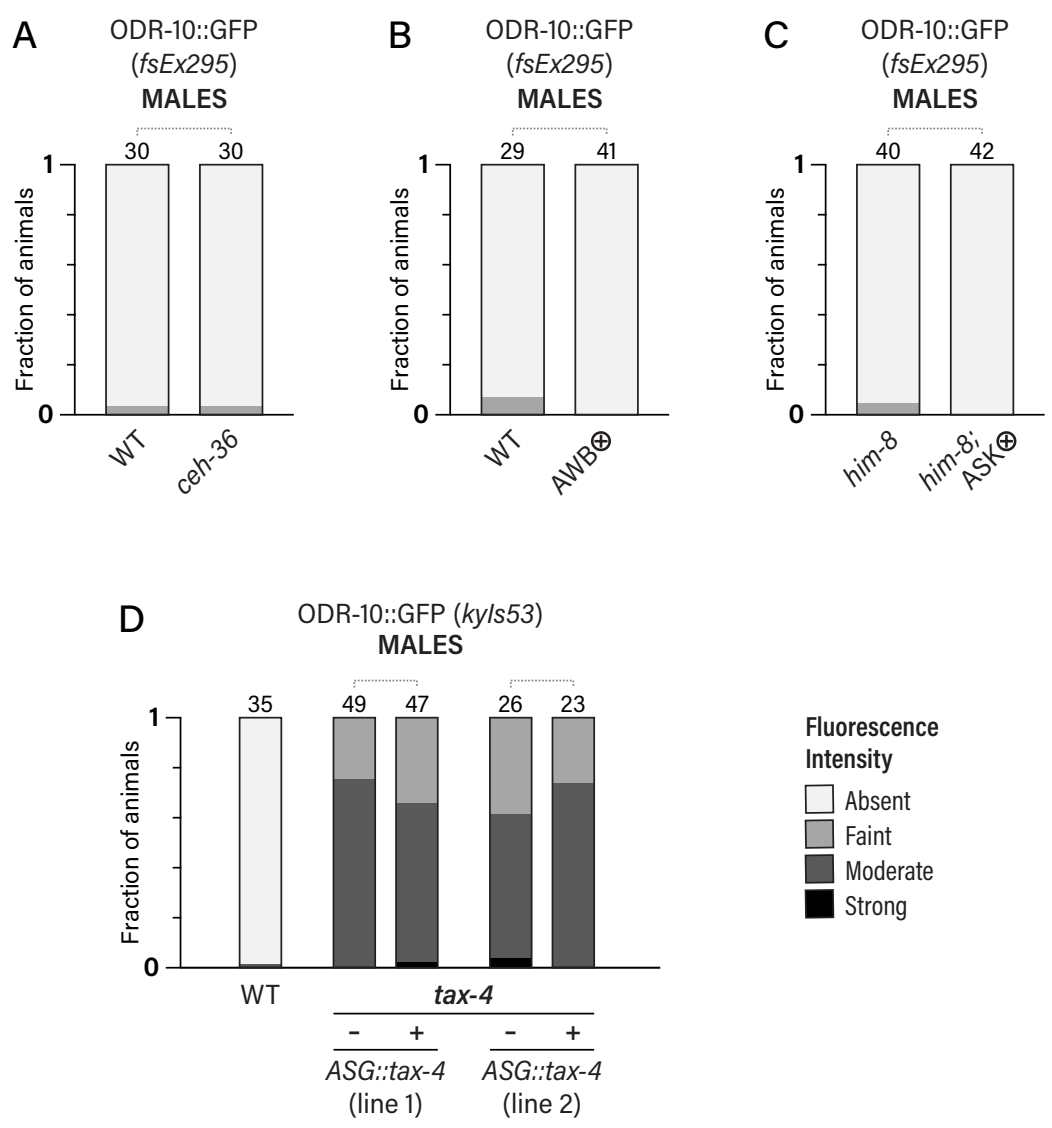

Figure S5. Related to Fig. 5. (A) ODR-10::GFP expression in WT and ceh-36 males (AWC genetic ablation). (B) ODR-10::GFP expression in WT and Pstr-1::mCasp1 males (AWB genetic ablation). (C) ODR-10::GFP expression in him-8 and him-8; Psra-9::mCasp1 males (ASK genetic ablation). (D) ODR-10::GFP (kyls53) expression in WT and tax-4 males with or without transgenes expressing tax-4 CDNA in ASG (Pops-1::tax4c). Results for two independent lines are shown. Dotted gray brackets indicate $p>0.05$. 